\title{
A Multi-Dimensional Central Limit Bound and its Application to the Euler Approximation of LÉVY-SDES
}

\author{
Xīlíng Zhāng *
}

16 June, 2017

\begin{abstract}
In the one-dimensional case Rio [17] gave a concise bound for the central limit theorem in the Vaserstein distances, which is a ratio between some higher moments and some powers of the variance. As a corollary, it gives an estimate for the normal approximation of the small jumps of Lévy processes, and Fournier [9] applied that to the Euler approximation of stochastic differential equations driven by the Lévy noise. It will be shown in this article that following Davie's idea in [7, one can generalise Rio's result to the multidimensional case, and have higher-order approximation via the perturbed normal distributions, if Cramér's condition and a slightly stronger moment condition are assumed. Fournier's result can then be partially recovered.
\end{abstract}

\section{Introduction}

Given 1$]$ equipped with a filtration $\left\{\mathscr{F}_{t}\right\}_{t \geqslant 0}$ generated by a $q_{1}$-dimensional Wiener process $\left\{W_{t}\right\}$ and an independent Poisson random measure $N(\mathrm{~d} z, \mathrm{~d} s)$ on $\mathbb{R}^{q} \backslash\{0\} \times[0, \infty)$ with intensity $\nu(\mathrm{d} z) \mathrm{d} s$. Consider the $q$-dimensional Lévy process on a bounded interval $[0, T]$ :

$$
Z_{t}=a t+B W_{t}+\int_{0}^{t} \int_{\mathbb{R}^{q} \backslash\{0\}} z \tilde{N}(\mathrm{~d} z, \mathrm{~d} s),
$$

where $\widetilde{N}(\mathrm{~d} z, \mathrm{~d} s)$ is the compensated Poisson measure. Assume the second moment of the Lévy measure $\int_{\mathbb{R}^{q} \backslash\{0\}}|z|^{2} \nu(\mathrm{d} z)<\infty$, where $|\cdot|$ denotes the modulus. For $x_{0} \in \mathbb{R}^{q}$ and a bounded Lipschitz function $\sigma: \mathbb{R}^{d} \rightarrow \mathbb{R}^{d \times q}$, consider the $d$-dimensional SDE driven by the Lévy process above:

$$
x_{t}=x_{0}+\int_{0}^{t} \sigma\left(x_{s-}\right) \mathrm{d} Z_{s}
$$

\footnotetext{
*School of Mathematics, The University of Edinburgh. e-mail: xiling.zhang@ed.ac.uk

${ }^{1}$ Throughout this article $\mathbb{Z}^{+}$and $\mathbb{N}$ denote the sets of positive and non-negative integers, respectively.
} 
For $h \in(0,1)$ and $t_{k}=k h, k=1, \cdots,[T / h]$, where $[\cdot]$ denotes the integer part, it is known that the standard Euler's approximation,

$$
X_{k+1}:=X_{k}+\sigma\left(X_{k}\right)\left(Z_{t_{k+1}}-Z_{t_{k}}\right), X_{0}=x_{0},
$$

converges with rate $1 / 2$ to the solution of (0.2) in mean-square as $h \rightarrow 0$ - see e.g. [14, [13] and [12. Although the increments $Z_{t_{k}}-Z_{t_{k-1}}$ are hard to generate, one may simply ignore the small jumps

$$
Z_{t}^{\epsilon}:=\int_{0}^{t} \int_{0<|z| \leqslant \epsilon} z \tilde{N}(\mathrm{~d} z, \mathrm{~d} s),
$$

for some $\epsilon \in(0,1)$, and show that the mean-square convergence rate is preserved. However, that is not a very economical way of simulation, as pointed out by Fournier [9]. Indeed, when the small jumps are completely ignored, the expected computational cost

$$
E_{\nu}(h)=O\left(h^{-1}+\nu(\{|z|>\epsilon)\}\right),
$$

can be considerably large. This happens, e.g., when the Lévy measure $\nu$ behaves like $\alpha$-stable near 0 , i.e. there exist $\tau>0$ and $\alpha \in(0,2)$ s.t. the following condition holds:

Assumption $\mathbf{H}(\tau, \alpha) . \nu(\mathrm{d} z) \simeq|z|^{-q-\alpha} \mathrm{d} z, \forall 0<|z| \leqslant \tau$.

The symbol $\simeq$ is used where both sides are bounded by each other up to a constant factor depending only on $q$. Given condition $\mathbf{H}(\tau, \alpha)$, the set of big jumps has measure $\nu(\{|z|>\epsilon\}) \simeq$ $\epsilon^{-\alpha}$, and one has to choose $\epsilon=h^{1 /(2-\alpha)}$ to ensure the order $1 / 2$ of mean-square convergence. As a result $E_{\nu}(h)=O\left(h^{-1}+h^{\alpha /(\alpha-2)}\right)$ explodes when $\alpha$ is close to 2 .

As a remedy, one may consider approximating the small jumps (0.3) with a normal random variable using the central limit theorem, on which some classical theorems can be found in several books such as [16] and [2]. Asmussen and Rosiński [1] adopted this idea and derived some Berry-Esseen bounds for the normal approximation of the small jumps $Z_{1}^{\epsilon}$; they also gave conditions for the weak convergence in the Skorohod space. But their method only works for $q=1$, and the Berry-Esseen-type bounds are not very useful for the strong $L^{p}$ approximation of Lévy-SDEs as they only concern the uniform distance between the c.d.f's. Aiming at the Euler approximation of (0.2), Fournier [9] proved that by adding this normal random variable to the Euler scheme the expected computational cost can be controlled (no explosion of $E_{\alpha}(h)$ near $\alpha=2$ ), while the $1 / 2$ convergence rate is still preserved. However, as pointed out himself, the method is also restricted to the case $q=1$.

Such a restriction of dimension only emerged at a key step in 9] (Corollary 4.2), borrowed from a result by Rio [17] (Corollary 4.2) on the central limit theorem. The latter ensures that, for a sequence of i.i.d., mean-0 random variables $2 X_{j} \in \mathbb{R}$ and $Y_{m}:=m^{-1 / 2} \sum_{j=1}^{m} X_{j}$ for any $m \in \mathbb{Z}^{+}$, there is an absolute constant $C$ s.t.

$$
\mathbb{W}_{2}\left(\mathbb{P}_{m}, \mathcal{N}(0, \operatorname{var} X)\right) \leqslant C\left(\frac{\mathbb{E}|X|^{4}}{\operatorname{var} X}\right)^{\frac{1}{2}} m^{-\frac{1}{2}},
$$

\footnotetext{
${ }^{2}$ When only the distribution of the $X_{j}$ 's is considered, the subscript $j$ is omitted for simplicity.
} 
where $\mathbb{P}_{m}$ denotes the distribution of $Y_{m}$ and $\mathbb{W}_{p}(\cdot, \cdot)$ is the $p$-Vaserstein (or "Wasserstein") distance. For probability measures $\mathbb{P}, \mathbb{Q}$ on $\mathbb{R}^{q}$, such a distance is defined by

$$
\mathbb{W}_{p}(\mathbb{P}, \mathbb{Q}):=\inf _{\pi \in \Pi(\mathbb{P}, \mathbb{Q})}\left(\int_{\mathbb{R}^{q} \times \mathbb{R}^{q}}|x-y|^{p} \pi(\mathrm{d} x, \mathrm{~d} y)\right)^{\frac{1}{p}},
$$

where $\Pi(\mathbb{P}, \mathbb{Q})$ is the set of all joint probability measures on $\mathbb{R}^{q} \times \mathbb{R}^{q}$ with marginal laws $\mathbb{P}$ and $\mathbb{Q}$. Rio [17] (Theorem 4.1) in fact only assumed the independence of $\left\{X_{j}\right\}$, but regarding central limit approximations and the simulation of Lévy processes one only considers the i.i.d. case. The constant $C$ in (0.4) would vary in $p$ for a bound in $\mathbb{W}_{p}$ and is later optimised in [18]. Apart from the restriction $q=1$, Rio's effective bounds only hold for $p \leqslant 4$. But this has been improved by Bobkov [3] (Theorem 1.1), allowing the $\mathbb{W}_{p^{-}}$convergence of order $O\left(m^{-1 / 2}\right.$ ) for any $p \geqslant 1$.

The dimensional restriction in Rio and Bobkov's results comes from the fact that when $q=1$, for $p \geqslant 1$ the $\mathbb{W}_{p}$ distance between two probability measures $\mathbb{P}, \mathbb{Q}$ on $\mathbb{R}$ is explicitly given (see Theorem 2.18 and Remarks 2.19 in [20]):

$$
\mathrm{W}_{p}(\mathbb{P}, \mathbb{Q})=\left(\int_{0}^{1}\left|F^{-1}(t)-G^{-1}(t)\right|^{p} \mathrm{~d} t\right)^{\frac{1}{p}},
$$

where $p \geqslant 1, F, G$ are the c.d.f's of $\mathbb{P}, \mathbb{Q}$, and $F^{-1}, G^{-1}$ are their generalised inverses, respectively. For $p=1$ there is a further equality $\mathbb{W}_{1}(\mathbb{P}, \mathbb{Q})=\int_{\mathbb{R}}|F(x)-G(x)| \mathrm{d} x$; in general there is no explicit formula for $q \geqslant 2$. However, if two probability distributions $\mathbb{P}$ and $\mathbb{Q}$ on $\mathbb{R}^{q}$ have densities $f$ and $g$, respectively, instead of the precise formula (0.5) there is the inequality

$$
\mathbb{W}_{p}(\mathbb{P}, \mathbb{Q}) \leqslant C_{p}\left(\int_{\mathbb{R}^{q}}|x|^{p}|f(x)-g(x)| \mathrm{d} x\right)^{\frac{1}{p}},
$$

for all $p \geqslant 1$, as a variant of Proposition 7.10 in [20].

This article presents an attempt to handle the normal approximation for the small jumps (0.3) for $q \geqslant 2$ using the bound (0.6), and give a positive answer to Fournier's question.

Davie 7] sketched an asymptotic approach via Edgeworth expansion of the density of $Y_{m}$, and proved (as a corollary to Proposition 2 therein) the rate $O\left(m^{-1 / 2}\right)$ under the assumption that all moments of $X$ are bounded. Moreover, he in fact showed a coupling between $Y_{m}$ and the normal distribution perturbed by polynomials. Section 1 of this article basically follows Davie's approach, but expounds detailed calculations and specify the range of $p$ and precisely how many moments of $X$ are needed - see Theorem 1.7 below.

The rate of convergence for the multi-dimensional central limit theorem has been studied using different methods. A strong result by Zaitsev (summarised as Theorem 2 in [22] and proved as Theorem 1.3 in [21]) gives a sharp Chernoff-type bound, and by Chebyshev's inequality the central limit theorem follows in a stronger sense: for independent $\left\{X_{j}\right\}$ each having identity covariance and independent standard Gaussian $\left\{\xi_{j}\right\}$ with partial sums $\Upsilon_{m}:=m^{-1 / 2} \sum_{j=1}^{m} \xi_{j}$, if the law of each $X_{j}$ satisfies certain analyticity conditions (see the definition of the class $\mathcal{A}_{q}(\tau)$ in [22]), then the distance $\max _{k \leqslant m}\left|Y_{k}-\Upsilon_{k}\right|$ is of order $O\left(m^{-1 / 2} \log m\right)$ in probability. 
The logarithmic factor emerges because the method is based on the dyadic approximation by Komlós, Major and Tusnády (KMT) [15]. The KMT method is much stronger than the usual central limit theorem since it considers the simultaneous approximation between $Y_{1}, Y_{2}, \cdots, Y_{m}$ and $\Upsilon_{1}, \Upsilon_{2}, \cdots, \Upsilon_{m}$. Einmahl [8] generalised the original KMT method to the multi-dimensional case, and Zaitsev's theorem [21] is an improved version of that, albeit it requires the local existence of the moment generating function.

Since the central limit theorem only concerns the coupling between $Y_{m}$ and $\Upsilon_{m}$, one should expect the $\log m$ factor to be removed as in the one-dimensional result of Rio. This has indeed been achieved by Bobkov [4] (Theorem 6.1) under the assumption that $\mathbb{E}|X|^{5}<\infty$; given only $\mathbb{E}|X|^{4}<\infty$, his result is weakened to $O\left(m^{-1 / 2}(\log m)^{q / 4-1}\right)$. It is worth mentioning here that, shortly after this article had been submitted, using Stein's method Bonis [5] (Theorem 8) managed to achieve the optimal rate $O\left(m^{-1 / 2}\right)$ given only $\mathbb{E}|X|^{4}<\infty$, which is a significant improvement. However, both approaches only work for $p=2$ since their arguments rely on some entropic transport inequalities for the $\mathbb{W}_{2}$ distance. In this special case (normal approximation for $Y_{m}$ in $\mathbb{W}_{2}$ ) the result derived in this article is not optimal, as it requires $\mathbb{E}|X|^{4+\tau}<\infty$ for some $\tau \in(0,1)$ and Cramér's condition $\varlimsup_{|s| \rightarrow \infty}|\mathbb{E} \exp (i s X)|<1$.

Nevertheless, given that $\mathbb{E}|X|^{6+\tau}<\infty$ and Cramér's condition, the result here would give a coupling for $Y_{m}$ of order $O\left(m^{-1}\right)$ in $\mathbb{W}_{p}$ for a positive even ingeter $p$, if one perturbs the normal distribution with a cubic Edgeworth polynomial. The Edgeworth expansion is used by Bobkov [3] (Corollary 9.2) in the one-dimensional case for higher-order approximations for $Y_{m}$, but in return Cramér's condition and some higher moments are needed. Theorem 1.7 here can be regarded as a generalisation of that.

In Section 2, the central limit bound in $\mathbb{W}_{p}$ is applied to the normal approximation for the small jumps (0.3). This is done by viewing $Z_{t}^{\epsilon}$ as a compound Poisson process, assuming Cramér's condition and that the Lévy measure $\nu$ is sufficiently singular at 0 (Theorem [2.1). A desired coupling $\mathbb{W}_{p}\left(Z_{t}^{\epsilon}, \sqrt{t} \mathcal{N}\left(0, \Sigma_{\epsilon}\right)\right)=O(\epsilon)$ is then achieved for $t=\epsilon$ and $\Sigma_{\epsilon}=\int_{0<|z| \leqslant \epsilon} z z^{\top} \nu(\mathrm{d} z)$, which covers the case $\mathbf{H}(\tau, \alpha)$. However, those assumptions can all be removed if one compromises for a suboptimal rate, as is proved in the appendices of Godinho's paper [10] (Proposition A.2), where only bounded jumps are considered. Again, there is a logarithmic factor because the proof directly uses the aforementioned result of Zaitsev.

Finally, the significance of using the Vaserstein distances instead of other ones is that, when generating numerical approximations for an SDE, the convergence in $\mathbb{W}_{p}$ is equivalent to the usual strong $L^{p}$-convergence. The reader is referred to the last section of [6] for a discussion on the contexts where such a substitution holds or fails. Unlike some of the results therein, the method to be introduced here is applicable to the simulation of stochastic flows defined by a Lévy SDE, since it only aims at a coupling for the increments $Z_{t_{k}}-Z_{t_{k-1}}$.

Throughout this article the generic positive constants $C$. and $c$. may change their values, with subscripts indicating their dependence of parameters. The notations $\lesssim$ and $\gtrsim$ indicate inequalities that hold with a factor $C_{q}$. The notation $\xi_{\Sigma}$ always stands for an $\mathbb{R}^{q}$-random variable following $\mathcal{N}(0, \Sigma)$. The symbol $|\cdot|$, depending on the object it acts on, stands for the modulus of vectors on $\mathbb{R}^{q}$, the absolute value for scalars, and the 1-norm of multi-indices on $\mathbb{N}^{q}$. For any $\rho \in \mathbb{N}^{q}$, it would be convenient to introduce the notation $|\rho|_{*}:=\rho_{1}+2 \rho_{2}+\cdots+q \rho_{q}$. 
In the context of matrices, $I$ stands for the identity matrix and $\|\cdot\|$ denotes any matrix norm.

\section{A Coupling for the Central Limit Theorem}

This section follows Davie's asymptotic approach via Edgeworth expansion briefly sketched in [7], and elaborates each step rigorously. The goal is to achieve a good $\mathbb{W}_{p}$ bound using (0.6), and for that one may first approximate the Fourier transform.

\subsection{Asymptotic Estimates of the Characteristic Function}

Let $q \in \mathbb{Z}^{+}$and $\left\{X_{j}\right\}_{j \geqslant 1}$ be a sequence of i.i.d. $\mathbb{R}^{q}$-random variables with mean 0 , covariance $\Sigma$ and characteristic function $\chi$, and define the weighted sum $Y_{m}=m^{-1 / 2} \sum_{i=1}^{m} X_{j}, m \in \mathbb{Z}^{+}$. Denote by $\psi_{m}$ and $\mathbb{P}_{m}$ the characteristic function and distribution of $Y_{m}$, respectively. Then one has asymptotic expansion (using the multi-index $\alpha \in \mathbb{N}^{q}$ ):

$$
\log \chi(s) \sim-\frac{1}{2} s \cdot \Sigma s+\sum_{|\alpha| \geqslant 3} \frac{i^{|\alpha|}}{\alpha !} \mu_{\alpha} s^{\alpha},
$$

where $\mu_{\alpha}=\mu_{\alpha}(X)=i^{-|\alpha|} \partial^{\alpha} \log \chi(0)$ is the $\alpha$-th cumulant of $X$. This gives a formal expansion for $\log \psi_{m}(z)=m \log \chi\left(m^{-1 / 2} z\right) \sim-\frac{1}{2} z \cdot \Sigma z+\sum_{|\alpha| \geqslant 3} \frac{i^{|\alpha|}}{\alpha !} m^{1-|\alpha| / 2} \mu_{\alpha} z^{\alpha}$, implying that

$$
\psi_{m}(z) \sim e^{-\frac{1}{2} z \cdot \Sigma z}\left(1+\sum_{k=1}^{\infty} m^{-\frac{k}{2}} P_{k}(z)\right),
$$

where $P_{k}(z)$ is a polynomial whose monomials have highest degree $3 k$ and lowest degree $k+2$, with coefficients bounded by $C_{k}\left(\mathbb{E}|X|^{k+2}\right)^{k}$ - see Lemma 7.1 in [2]. The inverse Fourier transform of (1.1) gives the Edgeworth expansion for the density $f_{m}$ of $Y_{m}$, if it exists. Detailed derivation for $q=1$ can also be found in [16] (Chapter VI).

In this section the shorthand notations $\varepsilon:=m^{-1 / 2}, \mathcal{P}_{\varepsilon, r}:=1+\sum_{k=1}^{r} \varepsilon^{k} P_{k}, \forall r \in \mathbb{Z}^{+}$, and $\mathcal{P}_{\varepsilon}:=\mathcal{P}_{\varepsilon, \infty}$ are used, and $\varepsilon$ and $m$ may be frequently interchanged. Denote by $\lambda_{1} \leqslant \cdots \leqslant \lambda_{q}$ the eigenvalues of $\Sigma$, and assume $\lambda_{1} \leqslant 1 \leqslant \lambda_{q}$ without loss of generality. Furthermore, $\forall M>0$ denote $\kappa_{M}:=1 \vee \mathbb{E}|X|^{M}$, then $\kappa_{M}^{1 / M}$ increases in $M$ by Hölder's inequality, and so does $\kappa_{M}$. By Lemma 6.3 in [2], $\left|\mu_{\alpha}\right| \leqslant C_{\alpha} \kappa_{|\alpha|}, \forall \alpha \in \mathbb{N}^{q}$.

Lemma 1.1. Suppose $\Sigma$ is non-singular and $\mathbb{E}|X|^{n+\tau}<\infty$ for a fixed integer $n \geqslant 3$ and $\tau \in(0,1)$. Let $\beta \in(0,1 / 3)$ and $\delta:=\min \left\{\lambda_{1} / \kappa_{3}, \kappa_{n}^{-1 / n} / 2\right\}$. Then for $|z| \leqslant m^{1 / 2} \delta, m \in$ $\mathbb{Z}^{+},\left|\psi_{m}(z)\right| \leqslant \exp \left(-\frac{1}{4} z \cdot \Sigma z\right) ;$ for $|z| \leqslant m^{\beta / 2}$ and $m>\max \left\{\left(\kappa_{3} / \lambda_{1}\right)^{3}, \kappa_{n+\tau}^{\max \{4,6 /(n(1-3 \beta))\}}\right\}$,

$$
\left|\psi_{m}(z)-e^{-\frac{1}{2} z \cdot \Sigma z} \mathcal{P}_{\varepsilon, n-2}(z)\right| \leqslant C_{n, \tau} \kappa_{n+\tau}^{n-2}\left(|z|^{n+1}+|z|^{3(n-1)}\right) e^{-\frac{1}{4} z \cdot \Sigma z} \varepsilon^{n-1} .
$$


Proof. First of all Taylor's theorem gives the identity

$$
\chi(s)=1-\frac{1}{2} s \cdot \Sigma s+\mathbb{E} \int_{0}^{1} \frac{1}{2} e^{i \theta(s \cdot X)}(1-\theta)^{2}(i s \cdot X)^{3} \mathrm{~d} \theta .
$$

Then for $|s| \leqslant \delta_{1}:=\lambda_{1} / \kappa_{3} \leqslant \sqrt{2 / \lambda_{q}}$, the inequality $\log u \leqslant u-1, \forall u>0$, implies that

$$
\begin{aligned}
\log |\chi(s)| & \leqslant \log \left(1-\frac{1}{2} s \cdot \Sigma s+\frac{1}{6} \mathbb{E}|X|^{3}|s|^{3}\right) \leqslant-\frac{1}{2} s \cdot \Sigma s+\frac{1}{6} \delta_{1} \mathbb{E}|X|^{3}|s|^{2} \\
& \leqslant-\frac{1}{2} s \cdot \Sigma s+\frac{1}{4} \lambda_{1}|s|^{2} \leqslant-\frac{1}{4} s \cdot \Sigma s,
\end{aligned}
$$

and the first claim $\left|\psi_{m}(z)\right| \leqslant \exp \left(-\frac{1}{4} z \cdot \Sigma z\right)$ holds for $|z| \leqslant m^{1 / 2} \delta_{1}$.

On the other hand, for $|s| \leqslant \kappa_{3}^{-1 / 3} / 2 \leqslant \lambda_{q}^{-1 / 2} / 2$, from (1.3) one sees that

$$
\operatorname{Re} \chi(s) \geqslant 1-\frac{1}{2} \lambda_{q}|s|^{2}-\frac{1}{6} \mathbb{E}|X|^{3}|s|^{3}>\frac{1}{2},
$$

and hence the principle branch of $\log \chi(s)$ is well-defined, and $|\chi(s)|>1 / 2$. For fixed $n \geqslant 3$, define, $\forall s \in \mathbb{R}^{q}$,

$$
S_{n}(s):=\sum_{|\alpha|=2}^{n} \frac{i^{|\alpha|}}{\alpha !} \mu_{\alpha} s^{\alpha}, T_{n}(s):=\sum_{|\alpha|=2}^{n} \frac{i^{|\alpha|}}{\alpha !} s^{\alpha} \mathbb{E} X^{\alpha}=\sum_{j=2}^{n} \frac{1}{j !} \mathbb{E}(i s \cdot X)^{j} .
$$

Then $\forall u \in \mathbb{R}$, using the inequality $\left|e^{i u}-1\right| \leqslant 2 \wedge|u| \leqslant 2^{1-\tau}|u|^{\tau}, \forall \tau \in(0,1)$, and the identity

$$
e^{i u}=\sum_{k=0}^{n} \frac{(i u)^{k}}{k !}+\frac{i^{n}}{(n-1) !} \int_{0}^{1}(1-\theta)^{n-1} u^{n}\left(e^{i \theta u}-1\right) \mathrm{d} \theta
$$

one deduces $\left|\chi(s)-1-T_{n}(s)\right| \leqslant C_{n, \tau} \kappa_{n+\tau}|s|^{n+\tau}$ by the substitution $u=s \cdot X$. Meanwhile one can write the following expansion (with Taylor remainder $R_{n}(s)$ ):

$$
\log \left(1+T_{n}(s)\right)=\sum_{l=1}^{n} \frac{(-1)^{l+1}}{l} T_{n}^{l}(s)+R_{n}(s)=S_{n}(s)+\widetilde{S}_{n}(s)+R_{n}(s),
$$

where $\widetilde{S}_{n}(s)$ is a polynomial of which each monomial has degree at least $n+1$. The fact that the first few terms agree with $S_{n}(s)$ is due to the relation between the cumulants $\mu_{\alpha}$ and the moments $\mathbb{E} X^{\alpha}$ - see Section 6 (page 46) in [2]. By the multinomial theorem, for $l=1, \cdots, n$ each monomial in $T_{n}^{l}(s)$ takes the form

$$
\sigma_{\rho, l}(s)=C_{n, l, \rho} \prod_{j=1}^{n-1}\left(\mathbb{E}(s \cdot X)^{j+1}\right)^{\rho_{j}}
$$

for some $\rho \in \mathbb{N}^{n-1},|\rho|=l$. Then the monomials $\widetilde{\sigma}_{\rho, l}(s)$ of $\widetilde{S}_{n}$ correspond to those with $\sum_{j=1}^{n-1}(j+1) \rho_{j}=|\rho|_{*}+l \geqslant n+1$. If one further chooses $\delta_{2}:=\kappa_{n}^{-1 / n} / 2<1$, then for $|s| \leqslant \delta_{2}$,

$$
\begin{aligned}
\left|\widetilde{\sigma}_{\rho, l}(s)\right| & \leqslant C_{n, l}|s|^{|\rho|_{*}+l} \prod_{j=1}^{n-1} \kappa_{j+1}^{\rho_{j}} \leqslant C_{n, l}|s|^{n+1} \kappa_{n}^{-\left(|\rho|_{*}+l-(n+1)\right) / n} \prod_{j=1}^{n-1} \kappa_{j+1}^{\left(|\rho|_{*}+l\right) /(j+1)} \\
& =C_{n, l}|s|^{n+1} \kappa_{n}^{(n+1) / n} \prod_{j=1}^{n-1}\left(\kappa_{n}^{-1 / n} \kappa_{j+1}^{1 /(j+1)}\right)^{|\rho|_{*}+l} \leqslant C_{n, l} \kappa_{n}^{1+1 / n}|s|^{n+1},
\end{aligned}
$$


where Hölder's inequality is used in the last step. Therefore $\left|\widetilde{S}_{n}(s)\right| \leqslant C_{n} \kappa_{n}^{1+1 / n}|s|^{n+1}$. Also notice that, for $|s| \leqslant \delta_{2}$ and $j=2, \cdots, n$, one has $|s|^{j-1} \kappa_{j} \leqslant \kappa_{n}^{1 / n}\left(\kappa_{n}^{-1 / n} \kappa_{j}^{1 / j}\right)^{j} \leqslant \kappa_{n}^{1 / n}$. This implies that $\left|T_{n}\right| \leqslant \sum_{j=2}^{n}|s|^{j-1} \kappa_{j}|s| / j ! \leqslant(e-2) \kappa_{n}^{1 / n}|s| / 2<1 / 2$, and that $\left|1+\theta T_{n}(x)\right| \geqslant 1 / 2$ for any $\forall \theta \in[0,1]$. Therefore

$$
\left|R_{n}(s)\right| \leqslant \int_{0}^{1}(1-\theta)^{n}\left|\frac{T_{n}(s)}{1+\theta T_{n}(s)}\right|^{n+1} \mathrm{~d} \theta \leqslant C_{n}\left|T_{n}(s)\right|^{n+1} \leqslant C_{n} \kappa_{n}^{1+1 / n}|s|^{n+1} .
$$

Thus $\left|\log \left(1+T_{n}(s)\right)-S_{n}(s)\right| \leqslant C_{n} \kappa_{n}^{1+1 / n}|s|^{n+1}$. Since $|\chi(s)| \wedge\left|1+T_{n}(s)\right| \geqslant 1 / 2$ for $|s|<\delta_{2}$, the triangle inequality implies that

$$
\left|\log \chi(s)-S_{n}(s)\right| \leqslant 2\left|\chi(s)-1-T_{n}(s)\right|+\left|\log \left(1+T_{n}(s)\right)-S_{n}(s)\right| \leqslant C_{n, \tau} \kappa_{n+\tau}^{1+1 / n}|s|^{n+1} .
$$

Returning to $\psi_{m}$, as $\log \psi_{m}(z)=\varepsilon^{-2} \log \chi(\varepsilon z)$, from the estimate above one has

$$
\left|\log \psi_{m}(z)-\varepsilon^{-2} S_{n}(\varepsilon z)\right| \leqslant C_{n, \tau} \varepsilon^{n-1}|z|^{n+1} \kappa_{n+\tau}^{1+1 / n} .
$$

Moreover, writing $U_{n}(z):=\frac{1}{2} z \cdot \Sigma z+\varepsilon^{-2} S_{n}(\varepsilon z)$, one can apply Taylor's theorem again to the exponential $\exp \left(U_{n}(z)\right)$ (recall the notation $\left.\mathcal{P}_{\varepsilon, \cdot}\right)$ :

$$
\begin{aligned}
\exp \left(\sum_{|\alpha|=3}^{n} \frac{i^{|\alpha|}}{\alpha !} \varepsilon^{|\alpha|-2} \mu_{\alpha} z^{\alpha}\right) & =1+U_{n}(z)+\frac{1}{2 !} U_{n}^{2}(z)+\cdots+\frac{1}{(n-2) !} U_{n}^{n-2}(z)+V(z) \\
& =1+\mathcal{P}_{\varepsilon, n-2}(z)+\widetilde{P}(z)+V(z),
\end{aligned}
$$

where $\widetilde{P}(z)=0$ for $n=3$ (i.e. $P_{1}(z)=U_{3}(z)$ contains all the cubic terms) and otherwise a polynomial of degree $n(n-2)$ with complex coefficients that contain products of the cumulants $\mu_{\alpha}$ up to $|\alpha|=n$ and powers of $\varepsilon$ at least $n-1$; the Taylor remainder $V(z)$ is given by

$$
V(z)=\frac{1}{(n-2) !} \int_{0}^{1}(1-\theta)^{n-2} U_{n}^{n-1}(z) e^{\theta U_{n}(z)} \mathrm{d} \theta .
$$

For $|z| \leqslant m^{1 / 6}=\varepsilon^{-1 / 3}$, one claims the following bound:

$$
|\widetilde{P}(z)| \leqslant C_{n} \kappa_{n}^{n-2} \varepsilon^{n-1}\left(|z|^{n+3}+|z|^{3(n-1)}\right) .
$$

This can be seen by checking the powers of $\varepsilon$ and $z$ in each $U_{n}^{l}(z), l=1, \cdots, n-2$. For each $l$, the multinomial theorem gives (with multi-indices $\rho \in \mathbb{N}^{n-2}, \alpha \in \mathbb{N}^{q}$ )

$$
U_{n}^{l}(z)=(-1)^{l} \sum_{|\rho|=l}\left(\begin{array}{l}
l \\
\rho
\end{array}\right)(i \varepsilon)^{|\rho|_{*}} \prod_{j=1}^{n-2}\left(\sum_{|\alpha|=j+2} \frac{1}{\alpha !} \mu_{\alpha} z^{\alpha}\right)^{\rho_{j}} .
$$

Then each monomial of $U_{n}^{l}(z)$ is bounded by $C_{n, l} \kappa_{n}^{l} \varepsilon^{|\rho|_{*}}|z|^{|\rho|_{*}+2 l}$, and the monomials $\widetilde{p}_{\rho, l}(z)$ of $\widetilde{P}(z)$ correspond to those with $|\rho|_{*} \geqslant n-1$ and $l \geqslant 2$. When $|\rho|_{*}+2 l \leqslant 3(n-1)$ the claim 
follows immediately from interpolating the powers of $|z|$; when $|\rho|_{*}+2 l>3(n-1)$, note that $|\rho|_{*}>|\rho|=l$, and so for $|z| \leqslant \varepsilon^{-1 / 3}$,

$$
\left|\widetilde{p}_{\rho, l}(z)\right| \leqslant C_{n, l} \kappa_{n}^{l} \varepsilon^{\frac{2}{3}\left(|\rho|_{*}-l\right)+n-1}|z|^{3(n-1)} \leqslant C_{n} \kappa_{n}^{n-2} \varepsilon^{n-1}|z|^{3(n-1)} .
$$

Regarding the Taylor remainder $V(z)$, notice that for $|z| \leqslant \varepsilon^{-\beta}, \forall \beta \in(0,1 / 3)$, and $\varepsilon<\kappa_{n}^{-1}$,

$$
\begin{aligned}
\left|U_{n}(z)\right| & \leqslant \sum_{j=1}^{n-2} \varepsilon^{j}|z|^{j+2} \kappa_{j+2} \leqslant \sum_{j=1}^{n-2} \varepsilon^{j-\beta(j-1)}|z|^{3} \kappa_{j+2} \leqslant \sum_{j=1}^{n-2} \varepsilon^{\frac{2}{3}(j-1)} \kappa_{n}^{(j+2) / n} \varepsilon|z|^{3} \\
& \leqslant \sum_{j=1}^{n-2} \kappa_{n}^{\frac{3}{n}+\left(\frac{1}{n}-\frac{2}{3}\right)(j-1)} \varepsilon|z|^{3} \leqslant(n-2) \kappa_{n}^{3 / n} \varepsilon|z|^{3},
\end{aligned}
$$

and furthermore $\left|U_{n}(z)\right| \leqslant(n-2) \kappa_{n}^{3 / n} \varepsilon^{1-3 \beta}$. Thus one arrives at

$$
|V(z)| \leqslant C_{n} \kappa_{n}^{3} \exp \left((n-2) \varepsilon^{1-3 \beta} \kappa_{n}^{3 / n}\right) \varepsilon^{n-1}|z|^{3(n-1)} .
$$

Combining with (1.5) one deduces, for $|z| \leqslant \varepsilon^{-\beta}$,

$$
\begin{aligned}
& \left|\psi_{m}(z)-e^{-\frac{1}{2} z \cdot \Sigma z} \mathcal{P}_{\varepsilon}^{(n-2)}(z)\right| \\
& \leqslant\left|e^{\log \psi_{m}(z)}-e^{-\frac{1}{2} z \cdot \Sigma z+U_{n}(z)}\right|+\left|e^{-\frac{1}{2} z \cdot \Sigma z+U_{n}(z)}-e^{-\frac{1}{2} z \cdot \Sigma z} \mathcal{P}_{\varepsilon, n-2}(z)\right| \\
& \leqslant\left|\psi_{m}(z)\right|\left|1-\exp \left(-\log \psi_{m}(z)-\frac{1}{2} z \cdot \Sigma z+U_{n}(z)\right)\right|+e^{-\frac{1}{2} z \cdot \Sigma z}(|\widetilde{P}(z)|+|V(z)|) \\
& \leqslant C_{n, \tau}\left|\psi_{m}(z)\right| \exp \left(\varepsilon^{2(n-2) / 3} \kappa_{n+\tau}^{1+1 / n}\right) \varepsilon^{n-1}|z|^{n+1} \kappa_{n+\tau}^{1+1 / n} \\
& \quad+C_{n} \kappa_{n}^{n-2} \exp \left((n-2) \varepsilon^{1-3 \beta} \kappa_{n}^{3 / n}\right) \varepsilon^{n-1}\left(|z|^{n+3}+|z|^{3(n-1)}\right) e^{-\frac{1}{2} z \cdot \Sigma z},
\end{aligned}
$$

where in the last step the inequality $\left|1-e^{u}\right| \leqslant e^{|u|}|u|, \forall u \in \mathbb{C}$, is used for the first term.

Now with $\delta:=\delta_{1} \wedge \delta_{2}$ fixed, for $m$ large one has $m^{\beta / 2}<m^{1 / 2} \delta$. Also, for fixed $\beta \in(0,1 / 3)$ and $\tau \in(0,1)$, one may further choose $m>\kappa_{n+\tau}^{3(1+1 / n) /(n-2)} \vee \kappa_{n}^{6 /(n(1-3 \beta))}$ s.t. the exponents in coefficients above are bounded by 1 . This is satisfied when $m>\kappa_{n+\tau}^{\max \{4,6 /(n(1-3 \beta))\}}$. For $m>\delta^{-3}>\delta^{2 /(\beta-1)}$ the first claim still holds, and so the second claim follows.

In order to bound the integral of the left-hand side term in (1.2) over all of $\mathbb{R}^{q}$, one may assume Cramér's condition:

$$
\varlimsup_{|s| \rightarrow \infty}|\chi(s)|<1,
$$

or equivalently, there exist $\rho>0$ and $\gamma \in(0,1)$ s.t. the following condition holds:

Assumption $\mathbf{C C}(\rho, \gamma) \cdot|\chi(s)| \leqslant \gamma, \forall|s| \geqslant \rho$.

As explained in [2] (page 207), if $\chi$ satisfies Cramér's condition, then $|\chi(s)|<1, \forall s \neq 0$; it is satisfied when $X$ has a density by the Riemann-Lebesgue theorem. Discrete distributions 
are excluded, but some singular and yet non-lattice distributions are also allowed, such as the distribution on the Cantor middle-third set that gives mass $2^{-j}$ to each interval on the $j$-th level.

Given the $X_{j}$ 's satisfying Cramér's condition, the following lemma shows that it is also satisfied for the weighted sum $Y_{m}$.

Lemma 1.2. Let $\chi$ satisfy $\mathbf{C C}(\rho, \gamma)$ with $\rho, \gamma$ explicitly known and $\delta \in(0, \rho \wedge 1)$. Then $\exists \bar{\gamma}=\bar{\gamma}(\rho, \gamma, \delta) \in(0,1)$ s.t. $\left|\psi_{m}(z)\right|<\bar{\gamma}^{m}$ for $|z|>m^{1 / 2} \delta$.

Proof. Let $N \in \mathbb{Z}^{+}$and write $\chi(N s)=|\chi(N s)| e^{i \theta_{1}}, \chi(s)=|\chi(s)| e^{i \theta_{0}}$, where $\theta_{1}, \theta_{0}$ depend on $s$. Then, with $F$ being the distribution of $X$, one gets $\int_{\mathbb{R}^{q}} \sin \left(s \cdot x-\theta_{0}\right) F(\mathrm{~d} x)=0$ and

$$
\begin{aligned}
1-|\chi(s)| & =\int_{\mathbb{R}^{q}}\left(1-\cos \left(s \cdot x-\theta_{0}\right)\right) F(\mathrm{~d} x)=\int_{\mathbb{R}^{q}} 2 \sin ^{2} \frac{1}{2}\left(s \cdot x-\theta_{0}\right) F(\mathrm{~d} x) \\
& \geqslant \frac{1}{N^{2}} \int_{\mathbb{R}^{q}} 2 \sin ^{2} \frac{N}{2}\left(s \cdot x-\theta_{0}\right) F(\mathrm{~d} x)=\frac{1}{N^{2}} \int_{\mathbb{R}^{q}}\left(1-\cos \left(N s \cdot x-N \theta_{0}\right)\right) F(\mathrm{~d} x),
\end{aligned}
$$

where the inequality $|\sin (N \phi)| \leqslant N|\sin \phi|, \forall N \in \mathbb{N}, \phi \in \mathbb{R}$, is used. Meanwhile,

$$
|\chi(N s)|=e^{-i \theta_{1}} \int_{\mathbb{R}^{q}} e^{i N s \cdot x} F(\mathrm{~d} x)=e^{i\left(N \theta_{0}-\theta_{1}\right)} \int_{\mathbb{R}^{q}} e^{i\left(N s \cdot x-N \theta_{0}\right)} F(\mathrm{~d} x),
$$

which implies

$$
\begin{aligned}
1-|\chi(s)| & \geqslant \frac{1}{N^{2}}-\frac{1}{N^{2}} \operatorname{Re} \int_{\mathbb{R}^{q}} e^{i\left(N s \cdot x-N \theta_{0}\right)} F(\mathrm{~d} x) \\
& \geqslant \frac{1}{N^{2}}-\frac{1}{N^{2}}\left|\int_{\mathbb{R}^{q}} e^{i\left(N s \cdot x-N \theta_{0}\right)} F(\mathrm{~d} x)\right|=\frac{1}{N^{2}}-\frac{1}{N^{2}}|\chi(N s)| .
\end{aligned}
$$

Choose $N=[(\rho+1) / \delta]>\rho / \delta$, then $|\chi(s)| \leqslant 1-(1-\gamma) \delta^{2} /(\rho+1)^{2}=: \bar{\gamma}$ for $\delta<|s|<\rho$. Clearly $\bar{\gamma} \geqslant \gamma$, and $\left|\psi_{m}(z)\right|=\left|\chi\left(m^{-1 / 2} z\right)\right|^{m}<\bar{\gamma}^{m}<1$ for $|z|>m^{1 / 2} \delta$.

From now on the following bounds will be frequently used: $\forall M, c>0$,

$$
\int_{\mathbb{R}^{q}}|x|^{M} e^{-c x \cdot \Sigma x} \mathrm{~d} x=\int_{\mathbb{R}^{q}}\left|\Sigma^{-\frac{1}{2}} y\right|^{M} e^{-c|y|^{2}} \operatorname{det}\left(\Sigma^{-\frac{1}{2}}\right) \mathrm{d} y \leqslant C_{q, c, M}(\operatorname{det} \Sigma)^{-\frac{1}{2}} \lambda_{1}^{-\frac{M}{2}},
$$

and

$$
\int_{\mathbb{R}^{q}}|x|^{M} \phi_{\Sigma}(x) \mathrm{d} x=C_{q} \int_{\mathbb{R}^{q}}\left|\Sigma^{\frac{1}{2}} y\right|^{M} e^{-\frac{1}{2}|y|^{2}} \mathrm{~d} y \leqslant C_{q, M} \lambda_{q}^{\frac{M}{2}}
$$

where the inverse and the square root of $\Sigma$ are well-defined since it is positive definite.

Although Cramér's condition gives some restriction on the law of $X$, it does not require the smoothness or the existence of the density $f_{m}$ of $Y_{m}$. In order to see how close the law of $Y_{m}$ is to the perturbed normal distributions from polynomial expansions, one may use a smoothing argument. Let $\widetilde{f}_{m}$ and $\widetilde{\psi}_{m}$ be the density and characteristic function of the mollified measure $\mathbb{P}_{m} * \theta_{m}$, where $\theta_{m}$ is a measure with smooth density, still denoted by $\theta_{m}$ or $\theta_{\varepsilon}$ :

$$
\theta_{\varepsilon}(x)=\varepsilon^{-q(n+1)} h\left(\varepsilon^{-n-1} x\right),
$$


for some function $0 \leqslant h \in C_{0}^{\infty}\left(\mathbb{R}^{q}\right)$ supported on the open unit ball and $\int_{\mathbb{R}^{q}} h(x) \mathrm{d} x=1$. Thus $\theta_{\varepsilon}$ is a probability density supported on $\left\{|x|<\varepsilon^{n+1}\right\}$. Write $\hat{h}$ and $\hat{\theta}_{\varepsilon}$ as their respective Fourier transforms.

Proposition 1.3. Under the assumptions in Lemma 1.1 and Lemma 1.2, for any integer $n \geqslant 3, \tau \in(0,1), \beta \in(0,1 / 3)$ and $m$ sufficiently large, it holds true that

$$
\int_{\mathbb{R}^{q}}\left|\widetilde{\psi}_{m}(z)-e^{-\frac{1}{2} z \cdot \Sigma z} \mathcal{P}_{\varepsilon, n-2}(z)\right| \mathrm{d} z \leqslant C_{q, n, \tau}(\operatorname{det} \Sigma)^{-\frac{1}{2}} \lambda_{1}^{-\frac{n-1}{2 \beta}} \kappa_{n+\tau}^{n-2} \varepsilon^{n-1} .
$$

Proof. Note that $\widetilde{\psi}_{m}=\psi_{m} \hat{\theta}_{\varepsilon}$, and for $|z| \leqslant m^{1 / 2} \delta$,

$$
\begin{aligned}
\left|\widetilde{\psi}_{m}(z)-\psi_{m}(z)\right| & =\left|\psi_{m}(z)\right|\left|\hat{\theta}_{\varepsilon}(z)-1\right| \leqslant\left|\psi_{m}(z)\right| \int_{|x|<\varepsilon^{n+1}}\left|e^{i z \cdot x}-1\right| \theta_{\varepsilon}(x) \mathrm{d} x \\
& \leqslant\left|\psi_{m}(z)\right||z| \varepsilon^{n+1} \leqslant \varepsilon^{n+1}|z| e^{-\frac{1}{4} z \cdot \Sigma z}
\end{aligned}
$$

and hence by Lemma 1.1 and triangle inequality,

$$
\left|\widetilde{\psi}_{m}(z)-e^{-\frac{1}{2} z \cdot \Sigma z} \mathcal{P}_{\varepsilon}^{(n-2)}(z)\right| \leqslant C_{n, \tau} \varepsilon^{n-1} \kappa_{n+\tau}^{n-2}\left(|z|^{n+1}+|z|^{3(n-1)}\right) e^{-\frac{1}{4} z \cdot \Sigma z},
$$

for $|z| \leqslant m^{\beta / 2}$. Also for all $z \in \mathbb{R}^{q}$,

$$
\begin{aligned}
\left|\hat{\theta}_{\varepsilon}(z)\right| & =\left|\int_{|x|<\varepsilon^{n+1}} e^{i z \cdot x} \theta_{\varepsilon}(x) \mathrm{d} x\right|=\left|\int_{|x|<\varepsilon^{n+1}} e^{i z \cdot x} \varepsilon^{-q(n+1)} h\left(\varepsilon^{-n-1} x\right) \mathrm{d} x\right| \\
& =\left|\int_{|y|<1} e^{i \varepsilon^{n+1} z \cdot y} h(y) \mathrm{d} y\right|=\left|\hat{h}\left(\varepsilon^{n+1} z\right)\right| \leqslant C_{q} \varepsilon^{-K(n+1)}|z|^{-K},
\end{aligned}
$$

for any $K>0$, since $h \in C_{0}^{\infty}\left(\mathbb{R}^{q}\right)$ with all the derivatives in $L^{1}\left(\mathbb{R}^{q}\right)$. One may choose $K=q+1$ for convenience and $\left|\widetilde{\psi}_{m}(z)\right| \leqslant \bar{\gamma}^{m} \min \left\{1, C_{q} \varepsilon^{-(q+1)(n+1)}|z|^{-q-1}\right\}$ for $|z|>m^{1 / 2} \delta$. For $|z| \leqslant m^{1 / 2} \delta$ one still has $\left|\widetilde{\psi}_{m}(z)\right| \leqslant \exp \left(-\frac{1}{4} z \cdot \Sigma z\right)$.

Given all the estimates for $\widetilde{\psi}_{m}(z)$ on different domains, one can split the integral in question into three parts:

$$
\begin{aligned}
\widetilde{I} & :=\int_{\mathbb{R}^{q}}\left|\widetilde{\psi}_{m}(z)-e^{-\frac{1}{2} z \cdot \Sigma z} \mathcal{P}_{\varepsilon}^{(n-2)}(z)\right| \mathrm{d} z \\
& =\left(\int_{|z| \leqslant m^{\beta / 2}}+\int_{m^{\beta / 2}<|z| \leqslant m^{1 / 2} \delta}+\int_{|z|>m^{1 / 2} \delta}\right)\left|\widetilde{\psi}_{m}(z)-e^{-\frac{1}{2} z \cdot \Sigma z} \mathcal{P}_{\varepsilon}^{(n-2)}(z)\right| \mathrm{d} z .
\end{aligned}
$$

Then by virtue of Lemma 1.1, Lemma 1.2.

$$
\begin{aligned}
\widetilde{I} & \leqslant C_{n, \tau} \kappa_{n+\tau}^{n-2} \varepsilon^{n-1} \int_{|z| \leqslant m^{\beta / 2}}\left(|z|^{n+1}+|z|^{3(n-1)}\right) e^{-\frac{1}{4} z \cdot \Sigma z} \mathrm{~d} z+\int_{m^{\beta / 2}<|z| \leqslant m^{\frac{1}{2}} \delta} e^{-\frac{1}{4} z \cdot \Sigma z} \mathrm{~d} z \\
& +\int_{|z|>m^{1 / 2} \delta} \bar{\gamma}^{m}\left(1 \wedge C_{q} \varepsilon^{-(q+1)(n+1)}|z|^{-q-1}\right) \mathrm{d} z+\int_{|z|>m^{\beta / 2}} e^{-\frac{1}{2} z \cdot \Sigma z}\left|\mathcal{P}_{\varepsilon, n-2}(z)\right| \mathrm{d} z .
\end{aligned}
$$


Use (1.6) for the first integral, combine the second and the fourth, and split the third into the set where $|z|$ is large and its complement to get $\left(\Lambda\right.$ denotes the Lebesgue measure on $\mathbb{R}^{q}$ )

$$
\begin{aligned}
\widetilde{I} \leqslant & C_{q, n, \tau}(\operatorname{det} \Sigma)^{-\frac{1}{2}} \lambda_{1}^{-\frac{3}{2}(n-1)} \kappa_{n+\tau}^{n-2} \varepsilon^{n-1}+\bar{\gamma}^{m} \Lambda\left(\left\{|z| \leqslant C_{q} \varepsilon^{-n-1}\right\}\right) \\
& +C_{q} \bar{\gamma}^{m} \varepsilon^{-(q+1)(n+1)} \int_{|z|>C_{q} \varepsilon^{-n-1}}|z|^{-q-1} \mathrm{~d} z+2 \int_{|z|>m^{\beta / 2}} e^{-\frac{1}{4} z \cdot \Sigma z}\left(1+\sum_{k=1}^{n-2} \varepsilon^{k}\left|P_{k}(z)\right|\right) \mathrm{d} z \\
\leqslant & C_{q, n, \tau}(\operatorname{det} \Sigma)^{-\frac{1}{2}} \lambda_{1}^{-\frac{3}{2}(n-1)} \kappa_{n+\tau}^{n-2} \varepsilon^{n-1}+C_{q} \bar{\gamma}^{m} \varepsilon^{-(q+1)(n+1)} \\
& +C_{q, n} \int_{|z|>m^{\beta / 2}} e^{-\frac{1}{4} z \cdot \Sigma z} \kappa_{n}^{n-2}\left(1+\sum_{k=1}^{n-2} \varepsilon^{k}|z|^{3 k}\right) \mathrm{d} z .
\end{aligned}
$$

The second term can be absorbed by the first term if $m$ is sufficiently large s.t. it satisfies the criterion of Lemma 1.1 and that

$$
\bar{\gamma}^{m} m^{\frac{1}{2}(q+1)(n+1)} \leqslant(\operatorname{det} \Sigma)^{-\frac{1}{2}} \lambda_{1}^{-\frac{3}{2}(n-1)} \kappa_{n+\tau}^{n-2} .
$$

For the third term, notice that $|z|>1$ and that $1<\varepsilon|z|^{1 / \beta}, \forall \beta \in(0,1 / 3)$. Thus

$$
\widetilde{I} \leqslant C_{q, n, \tau}(\operatorname{det} \Sigma)^{-\frac{1}{2}} \lambda_{1}^{-\frac{3}{2}(n-1)} \kappa_{n+\tau}^{n-2} \varepsilon^{n-1}+C_{q, n} \kappa_{n}^{n-2} \int_{\mathbb{R}^{q}} e^{-\frac{1}{4} z \cdot \Sigma z}\left(\varepsilon|z|^{1 / \beta}\right)^{n-1} \mathrm{~d} z,
$$

and the result follows from (1.6) again.

\subsection{Perturbed Normal Distributions}

Now given Proposition 1.3 , one can approximate the density $\widetilde{f}_{m}$ by the inverse Fourier transform $\mathcal{F}^{-1}$ of $\exp \left(-\frac{1}{2} z \cdot \Sigma z\right) \mathcal{P}_{\varepsilon}(z)$. Define, $\forall x \in \mathbb{R}^{q}$, the Edgeworth polynomials $\left\{Q_{k}\right\}$ by

$$
\phi_{\Sigma}(x) Q_{k}(x):=\mathcal{F}^{-1}\left\{e^{-\frac{1}{2} z \cdot \Sigma z} P_{k}(z)\right\}(x), \forall k \in \mathbb{Z}^{+},
$$

and accordingly $\mathcal{Q}_{\varepsilon, r}:=1+\sum_{k=1}^{r} \varepsilon^{k} Q_{k}, \forall r \in \mathbb{Z}^{+}$. Then each monomial of $Q_{k}$ has the same degree as that of $P_{k}$. In fact, if $\Sigma=\operatorname{diag}\left(\lambda_{1}, \cdots, \lambda_{q}\right)$, one can explicitly show that

$$
Q_{k}(x)=\sum_{|\alpha|=k+2}^{3 k}(-1)^{|\alpha|} b_{\alpha} \prod_{j=1}^{q} \lambda_{j}^{-\alpha_{j} / 2} H_{\alpha_{j}}\left(\lambda_{j}^{-1 / 2} x_{j}\right),
$$

where $b_{\alpha}=b_{\alpha}\left(\mu_{\beta}:|\beta| \leqslant k+2\right)$ is the real coefficient of $(i z)^{\alpha}$ in $P_{k}(z)$ satisfying $\left|b_{\alpha}\right| \leqslant \kappa_{k+2}^{k}$, and $H_{j}$ is the Hermite polynomial of degree $j$. See [16] (Chapter VI $\S 1$ ) for the precise values.

Remark 1.4. Since $\exp \left(-\frac{1}{2} z \cdot \Sigma z\right) \mathcal{P}_{\varepsilon, n-2}(z)$ and $\psi_{m}(z)$ have the same derivatives at 0 up to order $n$, the Edgeworth sum $\phi_{\Sigma} \mathcal{Q}_{\varepsilon, n-2}$ and $Y_{m}$ have the same moments up to order $n$.

For a positive-definite $q \times q$ matrix $\Sigma$, let $\mathscr{P}_{\Sigma}$ be the set of polynomials $S: \mathbb{R}^{q} \rightarrow \mathbb{R}$ s.t. $\int_{\mathbb{R}^{q}} S_{j}(x) \phi_{\Sigma}(x) \mathrm{d} x=0$ and $\mathscr{P}_{G}$ be the set of polynomials $U: \mathbb{R}^{q} \rightarrow \mathbb{R}^{q}$ s.t. $U=\nabla u$ for some 
polynomial $u: \mathbb{R}^{q} \rightarrow \mathbb{R}$. Furthermore let $\mathscr{P}_{\Sigma}^{\infty}$ be the set of sequences $\left(S_{1}, S_{2}, \cdots\right), S_{j} \in \mathscr{P}_{\Sigma}$ and $\mathscr{P}_{G}^{\infty}$ be the set of sequences $\left(U_{1}, U_{2}, \cdots\right), U_{j} \in \mathscr{P}_{G}$.

Given polynomials $U_{j}: \mathbb{R}^{q} \rightarrow \mathbb{R}^{q}, j=1, \cdots, k$, define $\mathbf{U}_{\varepsilon, k}(x):=x+\sum_{j=1}^{k} \varepsilon^{j} U_{j}(x), \forall \varepsilon>0$. Then for a $\xi_{\Sigma}$ following $\mathcal{N}(0, \Sigma)$, the random variable $\mathbf{U}_{\varepsilon, k}\left(\xi_{\Sigma}\right)$ is said to have a perturbed normal distribution, whose density can be formally expressed as

$$
\zeta_{\varepsilon, k}(y)=\operatorname{det}\left(\mathrm{DU}_{\varepsilon, k}^{-1}(y)\right) \phi_{\Sigma}\left(\mathbf{U}_{\varepsilon, k}^{-1}(y)\right)
$$

Davie [7] (Section 2) showed, using a recursive construction, that one can approximate $\zeta_{\varepsilon, k}(y)$ by $\phi_{\Sigma}(y) \mathcal{S}_{\varepsilon, l}(y):=\phi_{\Sigma}(y)\left(1+\sum_{j=1}^{l} \varepsilon^{j} S_{j}(y)\right)$ up to order $O\left(\varepsilon^{l+1}\right)$, where for each $j \leqslant l, S_{j}$ : $\mathbb{R}^{q} \rightarrow \mathbb{R}$ is a polynomial uniquely determined by $U_{1}, \cdots, U_{j}$ only. Since $l$ is arbitrary, for each $k$ the polynomials $U_{1}, \cdots, U_{k}$ uniquely determine a sequence $\left(S_{1}, S_{2}, \cdots\right)$, and hence the map $\mathfrak{S}_{\Sigma}:\left(U_{1}, U_{2} \cdots\right) \mapsto\left(S_{1}, S_{2}, \cdots\right)$ is well-defined. Moreover, each $S_{j} \in \mathscr{P}_{\Sigma}$ by Lemma 1 in [7].

A given sequence $\left(S_{1}, S_{2}, \cdots\right) \in \mathscr{P}_{\Sigma}^{\infty}$ can have several preimages under $\mathfrak{S}_{\Sigma}$. But according to Lemma 2 in [7], if one restricts $\mathfrak{S}_{\Sigma}$ on $\mathscr{P}_{G}^{\infty}$ then it is a bijection]. As is shown in the preceding paragraphs therein, this follows from the bijectivity of the linear map

$$
\mathscr{L}_{\Sigma}: \mathscr{P}_{G} \rightarrow \mathscr{P}_{\Sigma}, U(x) \mapsto \nabla \cdot U(x)-x \cdot \Sigma^{-1} U(x) .
$$

The preimages of the bijection $\mathfrak{S}_{\Sigma}$ are defined inductively in the following way: given a sequence $\left(S_{1}, S_{2}, \cdots\right) \in \mathscr{P}_{\Sigma}^{\infty}$, suppose $U_{1}, \cdots, U_{k} \in \mathscr{P}_{G}$ are found with $\mathfrak{S}_{\Sigma}\left(U_{1}, \cdots, U_{k}\right)=$ $\left(S_{1}, \cdots, S_{k}, \widetilde{S}_{k+1}, \cdots\right)$, then adding an additional $U_{k+1}$ gives a different sequence

$$
\mathfrak{S}_{\Sigma}\left(U_{1}, \cdots, U_{k}, U_{k+1}\right)=\left(S_{1}, \cdots, S_{k}, \widetilde{S}_{k+1}-\mathscr{L}_{\Sigma} U_{k+1}, \cdots\right) .
$$

This means that $U_{k+1} \in \mathscr{P}_{G}$ is determined by the equation $\widetilde{S}_{k+1}-\mathscr{L}_{\Sigma} U_{k+1}=S_{k+1}$. Writing $U_{k+1}=\nabla u_{k+1}$, one looks for a polynomial $u_{k+1}$ that solves the Hermite-type equation

$$
-\Delta u_{k+1}(x)+x \cdot \Sigma^{-1} \nabla u_{k+1}(x)=S_{k+1}(x)-\widetilde{S}_{k+1}(x), x \in \mathbb{R}^{q} .
$$

For the initial step set $\widetilde{S}_{1} \equiv 0$ and solve the PDE by induction on the degree of $u_{1}$; at each step, first compute $\widetilde{S}_{k+1}$ from $u_{1}, \cdots, u_{k}$ and then solve the PDE again by induction on the degree of $u_{k+1}$ - see similar arguments presented in the proof of Lemma 1 in [6].

The computation of $\widetilde{S}_{k+1}(x)$ can be done in the following formal way. First write

$$
\phi_{\Sigma}(x)=\zeta_{\varepsilon, k}\left(\mathbf{U}_{\varepsilon, k}(x)\right) \operatorname{det}\left(\mathrm{D} \mathbf{U}_{\varepsilon, k}(x)\right),
$$

by a change of variables. With $U_{j}=\nabla u_{j}, \mathrm{DU}_{\varepsilon, k}(x)=I+\sum_{j=1}^{k} \varepsilon^{j} \mathrm{D}^{2} u_{j}(x)$, and so the determinant above can be expressed as $1+\varepsilon v_{1}(x)+\cdots+\varepsilon^{q k} v_{q k}(x)$, where for each $l \leqslant q k, v_{l}$ is the sum of $\left(\partial_{i_{1} j_{1}}^{2} u_{1}\right)^{\rho_{1}} \cdots\left(\partial_{i_{k} j_{k}}^{2} u_{k}\right)^{\rho_{k}}$ over all the second derivatives and all multi-indices $\rho \in \mathbb{N}^{k}$ s.t. $|\rho|_{*}=l$. Then by formally writing $\zeta_{\varepsilon, k}(y)=\phi_{\Sigma}(y) \widetilde{\mathcal{S}}_{\varepsilon}(y)$ with $y=\mathbf{U}_{\varepsilon, k}(x)$ and

\footnotetext{
${ }^{3}$ This is motivated by Brenier's transport theorem for the quadratic cost - see Theorem 2.12 in 20 for the general statement and Lemma 5 in [7] for a special case.
} 


$$
\begin{aligned}
& \widetilde{\mathcal{S}}_{\varepsilon}(y)=1+\sum_{j=1}^{k} \varepsilon^{j} S_{j}(y)+\sum_{j=k+1}^{\infty} \varepsilon^{j} \widetilde{S}_{j}(y), \text { one can rearrange (1.14) to get } \\
& \begin{aligned}
1 & +\varepsilon S_{1}(y)+\cdots+\varepsilon^{k} S_{k}(y)+\varepsilon^{k+1} \widetilde{S}_{k+1}(y)+\cdots \\
& =\frac{\exp \left\{\sum_{j=1}^{k} \varepsilon^{j} x \cdot \Sigma^{-1} \nabla u_{j}(x)+\frac{1}{2} \sum_{j_{1}, j_{2}=1}^{k} \varepsilon^{j_{1}+j_{2}} \nabla u_{j_{1}}(x) \cdot \Sigma^{-1} \nabla u_{j_{2}}(x)\right\}}{1+\varepsilon v_{1}(x)+\cdots+\varepsilon^{q k} v_{q k}(x)} \\
& =1+\varepsilon T_{1}(x)+\varepsilon^{2} T_{2}(x)+\cdots,
\end{aligned}
\end{aligned}
$$

where the series on the right-hand side is obtained by multiplying out the Maclaurin series for $e^{z}$ and $1 /(1+z)$. Since differentiating a polynomial only changes its coefficients by a constant and reduces its degree, $\left|T_{k+1}(x)\right| \leqslant C_{q, k}\left\|\Sigma^{-1}\right\|^{k+1} \sum_{|\rho|_{*}=k+1}\left(1+\left|u_{1}(x)\right|\right)^{\rho_{1}} \cdots\left(1+\left|u_{k}(x)\right|\right)^{\rho_{k}}$.

On the left-hand side, each polynomial $S_{j}(y)$ with degree $d_{j} \geqslant 1$ can be expressed as $S_{j}(x)+$ $\varepsilon w_{j, 1}(x)+\cdots+\varepsilon^{d_{j} k} w_{j, d_{j} k}(x)$ by its Taylor expansion about $x$. Since all the derivatives of $S_{j}(x)$ have their norms bounded by $C_{q, j}\left(1+\left|S_{j}(x)\right|\right)$, one has, for each $j \leqslant k$ and $l \leqslant d_{j} k$, that $\left|w_{j, l}(x)\right| \leqslant C_{q, j, l} \sum_{s=1}^{l} \sum_{|\rho|_{*}=l}\left(1+\left|S_{j}(x)\right|\right)\left|U_{1}(x)\right|^{\rho_{1}} \cdots\left|U_{s}(x)\right|^{\rho_{s}}$. Thus, by expanding out $\widetilde{S}_{k+1}(y)$ in terms of $x$ and matching the coefficients of $\varepsilon^{k+1}$ on both sides, one gets

$$
\widetilde{S}_{k+1}(x)=T_{k+1}(x)-w_{k, 1}(x)-w_{k-1,2}(x)-\cdots-w_{2, k-1}(x)-w_{1, k}(x) .
$$

Although the calculation for $\widetilde{S}_{k+1}$ above is completely formal, it is equivalent to Davie's construction in [7] due to the uniqueness of the power series expansion. For a rigorous proof of such an approximation of $\zeta_{\varepsilon, k}$, the reader is referred to Proposition 1 in [7].

Remark 1.5. The set $\mathscr{P}_{G}$ is invariant under orthogonal transformation: given $U(x) \in \mathscr{P}_{G}$ and an orthogonal matrix $A$, the polynomial $G(x)=A^{-1} U(A x)$ also lies in $\mathscr{P}_{G}$.

To see this, notice that if $U(x)=\nabla u(x)$ and $A$ is a $q \times q$ matrix, then $g(x):=u(A x)$ has gradient $A^{\top} U(A x)$ and so $G(x)=\nabla u(A x)$ if $A$ is orthogonal.

The following lemma is a quantitative application of Proposition 1 in [7].

Lemma 1.6. The real polynomials $\left\{Q_{k}\right\}_{k=1}^{\infty}$ uniquely determine a sequence of polynomials $\left\{p_{k}\right\}_{k=1}^{\infty} \in \mathscr{P}_{G}^{\infty}$ s.t. $\forall r \in \mathbb{Z}^{+}$and $\varepsilon$ sufficiently small,

(i) $\left|p_{k}(x)\right| \leqslant C_{q, k} \lambda_{1}^{-5 k(k+2)} \lambda_{q}^{1+\frac{5}{2} k(k+2)} \kappa_{r+2}^{k^{2}}\left(1+|x|^{3 k}\right)$ for all $k=1, \cdots, r$ and $x \in \mathbb{R}^{q}$;

(ii) The random variable $\mathbf{p}_{\varepsilon, r}\left(\xi_{\Sigma}\right):=\xi_{\Sigma}+\sum_{k=1}^{r} \varepsilon^{k} p_{k}\left(\xi_{\Sigma}\right)$ has density $\zeta_{\varepsilon, r}(x)=\phi_{\Sigma}(x) \mathcal{Q}_{\varepsilon, r}(x)+$ $R_{\varepsilon, r}(x)$, where for any $M \geqslant 1$,

$$
\int_{\mathbb{R}^{q}}|x|^{M}\left|R_{\varepsilon, r}(x)\right| \mathrm{d} x \leqslant C_{q, r, M} \lambda_{1}^{-5(r+1)(r+2)} \lambda_{q}^{\frac{5}{2}(r+1)(r+3)+\frac{M}{2}} \kappa_{r+2}^{(r+1)^{2}} \varepsilon^{r+1} .
$$

Proof. First of all, the Edgeworth polynomials $\left\{Q_{k}\right\}$ defined by (1.11) are orthogonal to $\phi_{\Sigma}$ :

$$
\int_{\mathbb{R}^{q}} \phi_{\Sigma}(x) Q_{k}(x) \mathrm{d} x=\widehat{\phi_{\Sigma} Q_{k}}(0)=1 \cdot P_{k}(0)=0 .
$$


Thus $\left\{Q_{k}\right\} \in \mathscr{P}_{\Sigma}^{\infty}$, and hence $\left\{p_{k}\right\}:=\mathfrak{S}_{\Sigma}^{-1}\left(\left\{Q_{k}\right\}\right)$ gives the sequence sought after; for a fixed $r, p_{1}, \cdots, p_{r}$ are determined by $Q_{1}, \cdots, Q_{r}$ only. Moreover, if $\mathfrak{S}_{\Sigma}\left(p_{1}, \cdots, p_{r}\right)=$ $\left(Q_{1}, \cdots, Q_{r}, \widetilde{Q}_{r+1}, \cdots\right)$, then the density $\zeta_{\varepsilon, r}$ of $\mathbf{p}_{\varepsilon, r}\left(\xi_{\Sigma}\right)$ can be approximated by the expansion $\phi_{\Sigma}\left(\mathcal{Q}_{\varepsilon, r}+\varepsilon^{r+1} \widetilde{Q}_{r+1}\right)$ according to Proposition 1 in [7]. More precisely, $\forall M \geqslant 1$,

$$
\int_{\mathbb{R}^{q}}|x|^{M}\left|\zeta_{\varepsilon, r}(x)-\phi_{\Sigma}(x)\left(\mathcal{Q}_{\varepsilon, r}(x)+\varepsilon^{r+1} \widetilde{Q}_{r+1}(x)\right)\right| \mathrm{d} x \leqslant C_{q, r, M} K_{r}^{N_{r}} \varepsilon^{r+2},
$$

where $K_{r}$ is an upper bound for $\|\Sigma\|,\left\|\Sigma^{-1}\right\|$ and the absolute value of the coefficients of $p_{1}, \cdots, p_{r}$, and $N_{r}=N_{r}(q, M)>0$ is a constant depending on the maximum degree of $p_{1}, \cdots, p_{r}$. Then for $\varepsilon \leqslant K_{r}^{-N_{r}}$ this bound can be brought down to $C_{q, r, M} \varepsilon^{r+1}$, and it remains to find an upper bound for $\widetilde{Q}_{r+1}$ to derive the estimates in question.

For all $k \leqslant r$, write $p_{k}=\nabla u_{k}$ where $u_{k}$ satisfies (1.13) with $S_{k} \equiv Q_{k}$ and $\widetilde{S}_{k} \equiv \widetilde{Q}_{k}$. Assume that $\Sigma$ is diagonal. Then by (1.12), $\forall k, x$ one has $\left|Q_{k}(x)\right| \leqslant C_{q, k} \lambda_{1}^{-3 k} \kappa_{k+2}^{k}\left(1+|x|^{3 k}\right)$. Now one can bound the polynomials $\left\{\widetilde{Q}_{k}\right\}$ and $\left\{u_{k}\right\}$ inductively. For each $k \leqslant r-1$ suppose that (i) holds true for all $j \leqslant k$ :

$$
\left|u_{j}(x)\right| \leqslant C_{q, j} \lambda_{1}^{-5 j(j+2)} \lambda_{q}^{1+\frac{5}{2} j(j+2)} \kappa_{r+2}^{j^{2}}\left(1+|x|^{3 j}\right) .
$$

From the construction of $T_{k+1}$ and $\left\{w_{j, l}\right\}$ one sees that,

$$
\begin{aligned}
\left|T_{k+1}(x)\right| & \leqslant C_{q, k}\left\|\Sigma^{-1}\right\|^{k+1} \lambda_{1}^{-5 \sum^{*} j(j+2) \rho_{j}} \lambda_{q}^{k+1+\frac{5}{2} \sum^{*} j(j+2) \rho_{j}} \kappa_{r+2}^{\sum^{*} j^{2} \rho_{j}}\left(1+|x|^{\sum^{*} 3 j \rho_{j}}\right) \\
& \leqslant C_{q, k} \lambda_{1}^{-(k+1)(5 k+11)} \lambda_{q}^{(k+1)\left(\frac{5}{2} k+6\right)} \kappa_{r+2}^{(k+1)^{2}}\left(1+|x|^{3(k+1)}\right), \\
\left|w_{j, l}(x)\right| & \leqslant C_{q, j, l} \lambda_{1}^{-3 j-5 \sum^{\dagger} s(s+2) \rho_{s}} \lambda_{q}^{l+\frac{5}{2} \sum^{\dagger} s(s+2) \rho_{s}} \kappa_{r+2}^{j+\sum^{\dagger} s^{2} \rho_{s}}\left(1+|x|^{\left.3 j+\sum^{\dagger} 3 s \rho_{s}\right)}\right. \\
& \leqslant C_{q, j, l} \lambda_{1}^{-3 j-5 l(l+2)} \lambda_{q}^{l+\frac{5}{2} l(l+2)} \kappa_{r+2}^{j+l(l+1)}\left(1+|x|^{3(j+l)}\right),
\end{aligned}
$$

where $\sum^{*}$ denotes the summation over $j=1 \cdots, k$ and all multi-indices $\rho \in \mathbb{N}^{k}$ s.t. $|\rho|_{*}=$ $k+1$, and $\sum^{\dagger}$ denotes the summation over $s=1, \cdots, l$ and all $\rho \in \mathbb{N}^{l}$ s.t. $|\rho|_{*}=l$. Then $\left|\sum_{j+l=k+1} w_{j, l}(x)\right| \leqslant C_{q, k} \lambda_{1}^{-5(k+1)(k+2)} \lambda_{q}^{k\left(\frac{5}{2} k+6\right)} \kappa_{r+2}^{(k+1)^{2}}\left(1+|x|^{3(k+1)}\right)$, which is no more than (1.17), and hence by (1.15) $\widetilde{Q}_{k+1}$ has the same bound as (1.17).

For each $\alpha \in \mathbb{N}^{q}$, it is known that the Hermite-type polynomial

$$
H_{\alpha, \Sigma}(x)=\frac{1}{\sqrt{\alpha !}} \prod_{j=1}^{q} H_{\alpha_{j}}\left(\lambda_{j}^{-1 / 2} x_{j}\right)
$$

is the eigenfunction of the differential operator of the equation (1.13) corresponding to the eigenvalue $\nu_{\alpha}:=\sum_{j=1}^{q} \alpha_{j} \lambda_{j}^{-1} \leqslant|\alpha| / \lambda_{q}$. Since $\left\{H_{\alpha, \Sigma}\right\}$ form an orthonormal basis for the Hilbert space $L^{2}\left(\mathbb{R}^{q}, \phi_{\Sigma} \mathrm{d} \Lambda\right)$, the polynomial $u_{k+1}$ can be expressed as

$$
u_{k+1}(x)=\sum_{|\alpha| \leqslant 3(k+1)} c_{\alpha} \nu_{\alpha}^{-1} H_{\alpha, \Sigma}(x),
$$


where $c_{\alpha}=\int_{\mathbb{R}^{q}}\left(Q_{k+1}(z)-\widetilde{Q}_{k+1}(z)\right) H_{\alpha, \Sigma}(z) \phi_{\Sigma}(z) \mathrm{d} z$. Then by the Cauchy-Schwartz inequality and (1.7), the above estimate for $\widetilde{Q}_{k+1}$ implies that

$$
\begin{aligned}
\left|u_{k+1}(x)\right| & \leqslant C_{q, k} \sum_{|\alpha| \leqslant 3(k+1)} C_{\alpha}\left(\int_{\mathbb{R}^{q}}\left|Q_{k+1}(z)-\widetilde{Q}_{k+1}(z)\right|^{2} \phi_{\Sigma}(z) \mathrm{d} z\right)^{\frac{1}{2}} \lambda_{q} \lambda_{1}^{-\frac{|\alpha|}{2}}\left(1+|x|^{|\alpha|}\right) \\
& \leqslant C_{q, k} \lambda_{1}^{-(k+1)(5 k+11)-\frac{3}{2}(k+1)} \lambda_{q}^{(k+1)\left(\frac{5}{2} k+6\right)+\frac{3}{2}(k+1)+1} \kappa_{r+2}^{(k+1)^{2}}\left(1+|x|^{3(k+1)}\right) \\
& \leqslant C_{q, k} \lambda_{1}^{-5(k+1)(k+3)} \lambda_{q}^{1+\frac{5}{2}(k+1)(k+3)} \kappa_{r+2}^{(k+1)^{2}}\left(1+|x|^{3(k+1)}\right),
\end{aligned}
$$

which agrees with the induction hypothesis; the initial step for $u_{1}$ also holds true as $\widetilde{Q}_{1} \equiv 0$. Therefore the bound in (i) holds true for each $u_{k}$, and it holds true for its gradient $p_{k}$, too. The induction step also gives the bound (1.17) for $\widetilde{Q}_{r+1}$, and hence (ii) follows from the triangle inequality and (1.7) again.

For a general positive-definite $\Sigma$, one diagonalises it with an orthogonal matrix $A$ and applies the same arguments above to the scaled polynomials $p_{k}^{*}(x):=A^{\top} p_{k}(A x)$. By Remark 1.5 the $p_{k}^{*}$ 's still lie in $\mathscr{P}_{G}$, and the results still hold.

The proof above takes a compromise approach by introducing $\widetilde{Q}_{r+1}$ in (1.16): the condition " $\varepsilon$ sufficiently small" is not needed for Lemma 1.6, as Proposition 1 in [7] allows an $O\left(\varepsilon^{r+1}\right)$ estimate for $\int_{\mathbb{R}^{q}}|x|^{M}\left|\zeta_{\varepsilon, r}(x)-\phi_{\Sigma}(x) \mathcal{Q}_{\varepsilon, r}(x)\right| \mathrm{d} x$ for all $\varepsilon>0$. However, whilst practically $K_{r}=\lambda_{1}^{-5 r(r+2)} \lambda_{q}^{1+5 r(r+2) / 2} \kappa_{r+2}^{r^{2}}$ by (i), it is rather complicated to compute $N_{r}$ explicitly.

Before proceeding to the main result, given fixed parameters $\beta \in(0,1 / 3)$ and $\bar{\gamma}, \tau \in(0,1)$, it would be convenient to combine all the criteria for $\varepsilon$ together: for any integer $r \geqslant 3$ the statement " $m$ sufficiently large w.r.t. $r$ " or " $\varepsilon$ sufficiently small w.r.t. $r$ " refers to that $m>\kappa_{r+\tau}^{\max \{4,6 /(r(1-3 \beta))\}} \vee K_{r-3}^{2 N_{r-3}}$ with $K_{0}, N_{0}:=1$ and that (1.10) holds for $n=r$.

\subsection{Main Result and Some Special Cases}

Given Lemma 1.6, it will be shown in the following theorem that the normal distribution $\mathcal{N}(0, \Sigma)$ perturbed by the polynomials $\left\{p_{k}\right\}$ is close to the law $\mathbb{P}_{m}$ in the Vaserstein distances. The proof is a more detailed and quantitative version of what is exhibited in Section 4 in [7], and specifies the dependence on $\Sigma$ and certain moments of $X$.

Theorem 1.7. Suppose $\Sigma$ is non-singular and $\chi$ satisfies $\mathbf{C C}(\rho, \gamma)$. Fix an integer $n \geqslant 3$, an even integer $p \in 2 \mathbb{Z}^{+}$and $\beta \in(0,1 / 3)$. If $\mathbb{E}|X|^{p(n-2)+2+\tau}<\infty$ for some $\tau \in(0,1)$, then for $m$ sufficiently large w.r.t. $p(n-2)+2$,

$$
\mathbb{W}_{p}\left(Y_{m}, \mathbf{p}_{m, n-3}\left(\xi_{\Sigma}\right)\right) \leqslant C_{p, q, n, \tau} \Xi_{X} m^{-(n-2) / 2},
$$

where $\mathbf{p}_{m, n-3}$ is the polynomial defined by Lemma 1.6, and $\Xi_{X}$ is a constant depending on $p, n, \beta, \eta, \Sigma, \mathbb{E}|X|^{p(n-2)+1}$ and $\mathbb{E}|X|^{p(n-2)+2+\tau}$. 
Proof. Denote $r=p(n-2)+2$. Taking the inverse Fourier transform, Proposition 1.3 implies that for all $x \in \mathbb{R}^{q}$ and for $m$ sufficiently large w.r.t. $r$,

$$
\begin{aligned}
\left|F_{r-2}(\varepsilon, x)\right|: & =\left|\widetilde{f}_{m}(x)-\phi_{\Sigma}(x) \mathcal{Q}_{\varepsilon, r-2}(x)\right| \leqslant C_{q} \int_{\mathbb{R}^{q}}\left|\widetilde{\psi}_{m}(z)-e^{-\frac{1}{2} z \cdot \Sigma z} \mathcal{P}_{\varepsilon, r-2}(z)\right| \mathrm{d} z \\
& \leqslant C_{q, r, \tau}(\operatorname{det} \Sigma)^{-\frac{1}{2}} \lambda_{1}^{-\frac{r-1}{2 \beta}} \kappa_{r+\tau}^{r-2} \varepsilon^{r-1}
\end{aligned}
$$

The goal is to use the inequality (0.6) to bound the $\mathbb{W}_{p}$ distance, for which one first writes

$$
\int_{\mathbb{R}^{q}}|x|^{p}\left|F_{r-2}(\varepsilon, x)\right| \mathrm{d} x \leqslant \int_{\mathbb{R}^{q}}|x|^{p}\left(\tilde{f}_{m}(x)+\phi_{\Sigma}(x)\left|\mathcal{Q}_{\varepsilon, r-2}(x)\right|\right) \mathrm{d} x \leqslant I_{1}+2 I_{2}+I_{3},
$$

where, for any $\eta \in(0,1)$,

$$
\begin{aligned}
I_{1} & :=\int_{|x| \leqslant \varepsilon^{-\eta /(p+q)}}|x|^{p}\left|F_{r-2}(\varepsilon, x)\right| \mathrm{d} x, I_{2}:=\int_{|x|>\varepsilon^{-\eta /(p+q)}}|x|^{p} \phi_{\Sigma}(x)\left|\mathcal{Q}_{\varepsilon, r-2}(x)\right| \mathrm{d} x, \\
I_{3} & :=\int_{|x|>\varepsilon^{-\eta /(p+q)}}|x|^{p} F_{r-2}(\varepsilon, x) \mathrm{d} x .
\end{aligned}
$$

For any fixed $p \geqslant 2$ and $\eta \in(0,1)$, one finds, by virtue of (1.18),

$$
I_{1} \leqslant C_{q, r, \tau}(\operatorname{det} \Sigma)^{-\frac{1}{2}} \lambda_{1}^{-\frac{r-1}{2 \beta}} \kappa_{r+\tau}^{r-2} \varepsilon^{r-1-\eta}
$$

For the integral on the complement $\left\{x:|x|>\varepsilon^{-\eta /(p+q)}\right\}=\left\{1<\varepsilon|x|^{(p+q) / \eta}\right\}$, one gets

$$
\begin{aligned}
I_{2} & \leqslant \int_{|x|>\varepsilon^{-\eta /(p+q)}}|x|^{p} \phi_{\Sigma}(x) \kappa_{r}^{r-2}\left(1+\sum_{k=1}^{r-2} \varepsilon^{k}|x|^{3 k}\right) \mathrm{d} x \\
& \leqslant C_{r} \int_{|x|>\varepsilon^{-\eta /(p+q)}}|x|^{p} \phi_{\Sigma}(x) \kappa_{r}^{r-2} \varepsilon^{r-1}|x|^{\frac{p+q}{\eta}(r-1)} \mathrm{d} x \leqslant C_{r} \lambda_{q}^{\frac{p}{2}+\frac{p+q}{2 \eta}(r-1)} \kappa_{r}^{r-2} \varepsilon^{r-1},
\end{aligned}
$$

due to the fact that $(p+q) / \eta>3$ and (1.7). Also observe that

$$
I_{3} \leqslant \int_{\mathbb{R}^{q}}|x|^{p}\left(\widetilde{f}_{m}(x)-\phi_{\Sigma}(x) \mathcal{Q}_{\varepsilon, r-2}(x)\right) \mathrm{d} x+I_{1}=: I_{4}+I_{1},
$$

by the triangle inequality. In order to get a good estimate for $I_{4}$, first observe that $\forall p \geqslant 2$ by Rosenthal's inequality - see e.g. Lemma 1 in [11],

$$
\int_{\mathbb{R}^{q}}|x|^{p} \mathbb{P}_{m}(\mathrm{~d} x)=\mathbb{E}\left|Y_{m}\right|^{p}=m^{-\frac{p}{2}} \mathbb{E}\left|\sum_{j=1}^{m} X_{j}\right|^{p} \leqslant C_{p}\left(m^{1-\frac{p}{2}} \mathbb{E}|X|^{p}+\left(\mathbb{E}|X|^{2}\right)^{\frac{p}{2}}\right) .
$$

Also, from the construction of $\theta_{\varepsilon}$ (1.8) (now supported on $\left\{|x|<\varepsilon^{r+1}\right\}$ ),

$$
\int_{\mathbb{R}^{q}}|x|^{p} \theta_{\varepsilon}(\mathrm{d} x)=\int_{|y|<1}|y|^{p} \varepsilon^{p(r+1)} h(y) \mathrm{d} y<\varepsilon^{p(r+1)},
$$


by a change of variables. For an even $p \geqslant 4$, as $p<r$ observe that all the moments up to $p$ of the expansion $\phi_{\Sigma} \mathcal{Q}_{\varepsilon, r-2}$ match those of $Y_{m}$ by Remark 1.4, Hence by (1.20) and (1.21),

$$
\begin{aligned}
I_{4} & \leqslant \int_{\mathbb{R}^{q}} \int_{\mathbb{R}^{q}}\left(|x+y|^{p}-|x|^{p}\right) \mathbb{P}_{m}(\mathrm{~d} x) \theta_{\varepsilon}(\mathrm{d} y) \leqslant C_{p, q} \sum_{k=1}^{p} \int_{\mathbb{R}^{q}} \int_{\mathbb{R}^{q}}|x|^{p-k}|y|^{k} \mathbb{P}_{m}(\mathrm{~d} x) \theta_{\varepsilon}(\mathrm{d} y) \\
& \leqslant C_{p, q} \varepsilon^{r+1} \int_{\mathbb{R}^{q}}|x|^{p-1} \mathbb{P}_{m}(\mathrm{~d} x) \leqslant C_{p, q} \varepsilon^{r+1}\left(\varepsilon^{p-3} \mathbb{E}|X|^{p-1}+\lambda_{q^{2}}^{\frac{p-1}{2}}\right) ;
\end{aligned}
$$

for $p=2$ the bound is reduced to $C_{p, q} \varepsilon^{r+1}\left(\mathbb{E}|X|^{2}\right)^{1 / 2}$ by (1.20) and Hölder's inequality. Therefore, altogether one arrives at, for $p \leqslant r$,

$$
\int_{\mathbb{R}^{q}}|x|^{p}\left|F_{r-2}(\varepsilon, x)\right| \mathrm{d} x \leqslant C_{p, q, r, \tau}\left((\operatorname{det} \Sigma)^{-\frac{1}{2}} \lambda_{1}^{-\frac{r-1}{2 \beta}}+\lambda_{q}^{\frac{p}{2}+\frac{p+q}{2 \eta}(r-1)}\right) \kappa_{r+\tau}^{r-2} \varepsilon^{r-1-\eta} .
$$

Finally by the triangle inequality one removes the $(r-2)$-th term in $\mathcal{Q}_{\varepsilon, r-2}$ :

$$
\int_{\mathbb{R}^{q}}|x|^{p}\left|F_{r-2}(\varepsilon, x)\right| \mathrm{d} x \geqslant \int_{\mathbb{R}^{q}}|x|^{p}\left|F_{r-3}(\varepsilon, x)\right| \mathrm{d} x-C_{q, r} \varepsilon^{r-2} \kappa_{r}^{r-2} \int_{\mathbb{R}^{q}}|x|^{p}\left(|x|^{r}+|x|^{3(r-2)}\right) \phi_{\Sigma} \mathrm{d} x,
$$

and uses (1.7) again to deduce the following estimate for the Edgeworth approximation:

$$
\int_{\mathbb{R}^{q}}|x|^{p}\left|F_{r-3}(\varepsilon, x)\right| \mathrm{d} x \leqslant C_{p, q, r}\left((\operatorname{det} \Sigma)^{-\frac{1}{2}} \lambda_{1}^{-\frac{r-1}{2 \beta}}+\lambda_{q}^{\frac{p}{2}+\frac{p+q}{2 \eta}(r-1)}\right) \kappa_{r+\tau}^{r-2} \varepsilon^{r-2} .
$$

Since the smooth measure $\theta_{\varepsilon}$ is also supported on $\left\{x:|x|<\varepsilon^{r-2}\right\}$, the estimate above implies that the Edgeworth polynomials $\left\{Q_{k}\right\} \in \mathscr{P}_{\Sigma}$ form an $\mathcal{A}_{\Sigma}$-sequence for the family of probability measures $\left\{\mathbb{P}_{m}\right\}$ - see Definition 1 in [7]. Then one can extend the expansion $\mathcal{Q}_{\varepsilon, r-3}$ to a larger value of $r$ and take the $p$-th root to get a $\mathbb{W}_{p}$ estimate, as in done in the proof of Theorem 4 in [7].

With the polynomials $\left\{p_{k}\right\}=\mathfrak{S}_{\Sigma}^{-1}\left(\left\{Q_{k}\right\}\right)$ and $\mathbf{p}_{\varepsilon, r-3}, R_{\varepsilon, r-3}$ defined as in Lemma 1.6, using the triangle inequality and the inequality $(\overline{0.6})$, one can deduce the following estimate for an integer $n \geqslant 3$ by replacing $r=p(n-2)+2$ in the estimate:

$$
\begin{aligned}
& \mathbb{W}_{p}\left(Y_{m}, \mathbf{p}_{\varepsilon, p(n-2)-1}\left(\xi_{\Sigma}\right)\right) \leqslant C_{p}\left(\int_{\mathbb{R}^{q}}|x|^{p}\left|F_{p(n-2)-1}\right| \mathrm{d} x+\int_{\mathbb{R}^{q}}|x|^{p}\left|R_{\varepsilon, p(n-2)-1}(x)\right| \mathrm{d} x\right)^{\frac{1}{p}} \\
& \leqslant C_{p, q, n, \tau}\left((\operatorname{det} \Sigma)^{-\frac{1}{2 p}} \lambda_{1}^{-\frac{1}{2 \beta}\left(n-2+\frac{1}{p}\right)}+\lambda_{q}^{\frac{1}{2}+\frac{p+q}{2 \eta}\left(n-2+\frac{1}{p}\right)}\right) \kappa_{p(n-2)+2+\tau}^{n-2} \varepsilon^{n-2} \\
& \quad+C_{p, q, n} \lambda_{1}^{-5(n-2)(p(n-2)+1)} \lambda_{q}^{\frac{1}{2}+\frac{5}{2}(n-2)(p(n-2)+1)} \kappa_{p(n-2)+1}^{p(n-2)^{2}} \varepsilon^{n-2},
\end{aligned}
$$

whilst the excess terms from $n-2$ to $p(n-2)-1$ can be handled by (i) of Lemma 1.6 and (1.7) again:

$$
\begin{aligned}
\mathbb{W}_{p}\left(\mathbf{p}_{\varepsilon, p(n-2)-1}\left(\xi_{\Sigma}\right), \mathbf{p}_{\varepsilon, n-3}\left(\xi_{\Sigma}\right)\right) & \leqslant C_{p}\left(\mathbb{E}\left|\sum_{k=n-2}^{p(n-2)-1} \varepsilon^{k} p_{k}\left(\xi_{\Sigma}\right)\right|^{p}\right)^{\frac{1}{p}} \\
& \leqslant C_{p, q, n} \lambda_{1}^{-5 p^{2}(n-2)^{2}+5} \lambda_{q}^{\frac{5}{2} p^{2}(n-2)^{2}+\frac{3}{2} p(n-2)-3} \kappa_{p(n-2)+1}^{(p(n-2)-1)^{2}} \varepsilon^{n-2} .
\end{aligned}
$$

Thus the claimed result follows from the triangle inequality. 
Remark 1.8. The number of moments of $X$ needed for Theorem 1.7 is independent of the dimension $q$.

The optimal result for the central limit theorem for $q=1$ is already given in [3], which is not fully recovered by Theorem 1.7 as the inequality (0.6) is rather crude compared to (0.5). For $q \geqslant 2$, Theorem 1.7 immediately implies the following (by choosing $n=3$ ):

Corollary 1.9. Suppose the i.i.d. random variables $\left\{X_{j}\right\}$ have non-singular covariance $\Sigma$ and satisfy $\mathbf{C C}(\rho, \gamma)$, and let $p \in 2 \mathbb{Z}^{+}$. If $\mathbb{E}|X|^{p+2+\tau}<\infty$ for some $\tau \in(0,1)$, then by taking e.g. $\beta=1 / 6, \eta=1 / 2$, the following holds for $m$ sufficiently large w.r.t. $p+2$ :

$$
\begin{aligned}
\mathbb{W}_{p}\left(Y_{m}, \xi_{\Sigma}\right) \leqslant & C_{p, q, \tau}\left((\operatorname{det} \Sigma)^{-1 /(2 p)} \lambda_{1}^{-3(1+1 / p)}+\lambda_{q}^{(p+q)(1+1 / p)+1 / 2}\right) \kappa_{p+2+\tau} m^{-1 / 2} \\
& +C_{p, q} \lambda_{1}^{-5 p^{2}+5} \lambda_{q}^{\left(5 p^{2}+3 p\right) / 2-3} \kappa_{p+1}^{2 \vee(p-1)^{2}} m^{-1 / 2}
\end{aligned}
$$

As mentioned in the introduction, for the special case $p=2$ this corollary is weaker than the results of Bobkov [4] and Bonis [5]. Although the condition $\mathbb{E}|X|^{4+\tau}<\infty$ is slightly better than that of Bobkov, he does not require Cramér's condition as he aimed at estimating the relative entropy $\mathbb{H}\left(\mathbb{P}_{m} \| \mathcal{N}(0, \Sigma)\right)$, which in turn gives a bound for the $\mathbb{W}_{2}$ distance according to Talagrand's transport inequality [19]. On the other hand, Bonis' optimal result relies on a differential estimate for the $\mathbb{W}_{2}$ distance in terms of the Fisher information. As the $\mathbb{W}_{2}$ distance enjoys those special properties, the inequality (0.6) does not provide a sharp bound.

However, Theorem 1.7 can potentially give higher-order convergence if one considers a nontrivial expansion $(n>3)$. For example, when choosing $n=4$, one gets a rate $O\left(m^{-1}\right)$ under Cramér's condition and that $\mathbb{E}|X|^{6+\tau}<\infty$. The task is to find the polynomial $p_{1}$ using the method described in the discussion before Lemma 1.6. given $Q_{1}(x)$ defined in (1.12), one looks for the unique (up to an additive constant) polynomial solution $u_{1}: \mathbb{R}^{q} \rightarrow \mathbb{R}$ satisfying (1.13) for the initial step:

$$
-\Delta u_{1}(x)+x \cdot \Sigma^{-1} \nabla u_{1}(x)=Q_{1}(x) .
$$

To illustrate that consider the simplest case where $q=2$ and $\Sigma=I$. The cubic polynomial $6 i P_{1}(z)=\mu_{(3,0)} z_{1}^{3}+3 \mu_{(2,1)} z_{1}^{2} z_{2}+3 \mu_{(1,2)} z_{1} z_{2}^{2}+\mu_{(0,3)} z_{2}^{3}$ gives

$$
6 Q_{1}(x)=\mu_{(3,0)} H_{3}\left(x_{1}\right)+3 \mu_{(2,1)} H_{2}\left(x_{1}\right) H_{1}\left(x_{2}\right)+3 \mu_{(1,2)} H_{1}\left(x_{1}\right) H_{2}\left(x_{2}\right)+\mu_{(0,3)} H_{3}\left(x_{2}\right) .
$$

Notice that $x \cdot \nabla u(x)=k u(x)$ for any monomial $u$ of degree $k$, and so the polynomial solution to the PDE above is cubic with no quadratic terms. Then using the property $H_{j}^{\prime}=j H_{j-1}$ and matching the coefficients on both sides of the equation, one gets

$$
\begin{aligned}
u_{1}(x)= & \frac{1}{18} \mu_{(3,0)} H_{3}\left(x_{1}\right)+\frac{1}{6} \mu_{(2,1)} H_{2}\left(x_{1}\right) H_{1}\left(x_{2}\right)+\frac{1}{6} \mu_{(1,2)} H_{1}\left(x_{1}\right) H_{2}\left(x_{2}\right)+\frac{1}{18} \mu_{(0,3)} H_{3}\left(x_{2}\right) \\
& +\frac{1}{3}\left(\mu_{(3,0)}+\mu_{(1,2)}\right) H_{1}\left(x_{1}\right)+\frac{1}{3}\left(\mu_{(0,3)}+\mu_{(2,1)}\right) H_{1}\left(x_{2}\right)+C
\end{aligned}
$$

and hence the perturbing polynomial $p_{1}=\nabla u_{1}$ is found. 
Under certain stronger conditions, one can also obtain higher-order convergence without specifying the perturbing polynomials $p_{k}$. For $q=1$, Bobkov [3] (Theorem 1.3) proved that if $\mathbb{E} X^{k}=\mathbb{E} \xi_{\Sigma}^{k}$ for $k=1, \cdots, n-1,3 \leqslant n \in \mathbb{Z}^{+}$, and $\mathbb{E}|X|^{p(n-2)+2}<\infty$, then under Cramér's condition one has $\mathbb{W}_{p}\left(Y_{m}, \xi_{\Sigma}\right)=O\left(m^{-(n-2) / 2}\right)$. This can be readily generalised to $q \geqslant 2$ by Theorem 1.7 if the first $n-1$ moments match those of $\mathcal{N}(0, \Sigma)$, the cumulants $\mu_{\alpha}(X)=\mu_{\alpha}\left(\xi_{\Sigma}\right)=0$ for all $|\alpha|=3, \cdots, n-1$, implying that $P_{k}=Q_{k} \equiv 0$. This immediately implies that $\mathcal{L}_{\Sigma} p_{k} \equiv 0$ in (1.13) for all $k=1, \cdots, n-3$, forcing $p_{k} \equiv 0$. Therefore one asserts the following:

Corollary 1.10. Suppose the i.i.d. random variables $\left\{X_{j}\right\}$ with non-singular covariance $\Sigma$ satisfy Cramér's condition and let $p \in 2 \mathbb{Z}^{+}$. If $\exists 3 \leqslant n \in \mathbb{Z}^{+}$s.t. $\mathbb{E} X^{\alpha}=\mathbb{E} \xi_{\Sigma}^{\alpha}$ for all $|\alpha|=$ $1, \cdots, n-1$, and $\mathbb{E}|X|^{p(n-2)+2+\tau}<\infty$ for some $\tau \in(0,1)$, then $\mathbb{W}_{p}\left(Y_{m}, \xi_{\Sigma}\right)=O\left(m^{-(n-2) / 2}\right)$ for $m$ sufficiently large w.r.t. $p(n-2)+2$.

\section{Application to the Euler Approximation of Lévy SDEs}

Consider the $d$-dimensional SDE (0.2) driven by a $q$-dimensional Lévy process (0.1). Assume that the Lévy measure $\nu$ has finite second moment, and the function $\sigma: \mathbb{R}^{d} \rightarrow \mathbb{R}^{d \times q}$ is bounded and Lipschitz. It will be shown in this section that the $q$-dimensional small jumps (0.3) can also be approximated by a normal random variable with rate 1 while the computational cost $E_{\nu}(h)$ remains controlled for $\nu$ satisfying certain stable-like conditions, in particular $\mathbf{H}(\tau, \alpha)$.

\subsection{Normal Approximation of Small Jumps via Central Limit Bound}

The way both Fournier [9] and Godinho [10] applied the central limit theorem for the small jumps $Z_{t}^{\epsilon}$ is to split the time interval into $m$ subdivisions and view $Z_{t}^{\epsilon}$ as the sum of the i.i.d. random variables $\int_{(j-1) t / m}^{j t / m} \int_{0<|z| \leqslant \epsilon} z \widetilde{N}(\mathrm{~d} z, \mathrm{~d} s), j=1, \cdots, m$. Here an alternative approach is considered: one may decompose the range of the jumps $\{0<|z| \leqslant \epsilon\}$ into countably many annuli and represent the small jumps as a sum:

$$
Z_{t}^{\epsilon}=\sum_{r=r_{0}}^{\infty} \int_{0}^{t} \int_{\Omega_{r}} z \widetilde{N}(\mathrm{~d} z, \mathrm{~d} s)=: \sum_{r=r_{0}}^{\infty} V_{t}^{r},
$$

where $\Omega_{r}=\left\{2^{-r-1}<|z| \leqslant 2^{-r}\right\}$ and $r_{0}=-\log _{2} \epsilon>0$. Assume $\nu$ to be $\sigma$-finite and denote $\nu_{r}:=\nu\left(\Omega_{r}\right)$. By the Lévy-Itō decomposition one knows that each $V_{t}^{r}$ is a compensated compound Poisson process:

$$
V_{t}^{r}=\sum_{j=1}^{N_{t}^{r}} X_{j}^{r}-t \nu_{r} \mathbb{E} X_{j}^{r},
$$

where $\left\{X_{j}^{r}\right\}$ are i.i.d. random variables bounded within $\Omega_{r}$ and $N_{t}^{r}$ follows $\operatorname{Poi}\left(t \nu_{r}\right)$.

Instead of directly working with $V_{t}^{r}$, one may first consider a general compound Poisson process $V_{t}$ of the form (2.2) with $N_{t} \sim \operatorname{Poi}(t \mu)$ and the jumps $X_{j}$ on the interval [0,1]. 
Expecting $\mu$ to be large, one can write

$$
Y=\mu^{-\frac{1}{2}} V_{1}=\mu^{-\frac{1}{2}} \sum_{j=1}^{N_{1}} X_{j}-\mu^{\frac{1}{2}} \mathbb{E} X_{j},
$$

and approximate it by Edgeworth-type polynomials using the same recipe just as before.

Let $\psi$ and $\chi$ be the characteristic functions of $Y$ and the $X_{j}$ 's, respectively, and $\Sigma_{X}$ be the covariance of $X$, with eigenvalues $\lambda_{1, X} \leqslant \cdots \leqslant \lambda_{q, X}$, and similarly $\kappa_{M, X}=1 \vee \mathbb{E}|X|^{M}, \forall M>$ 0 . Then one has the following simple relation between the distributions of $X$ and $Y$ :

$$
\psi(z)=\exp \left\{\mu\left(\chi\left(\mu^{-\frac{1}{2}} z\right)-1\right)-i \mu^{\frac{1}{2}} z \cdot \mathbb{E} X\right\} .
$$

Given this convenient expression, instead of taking the logarithm one may directly apply Taylor expansion to $\chi$ and have, instead of (1.1), a formal expansion

$$
\psi(z) \sim e^{-\frac{1}{2} z \cdot \Sigma_{X} z}\left(1+\sum_{k=1}^{\infty} \mu^{-\frac{k}{2}} P_{k}(z)\right),
$$

whose $(n-2)$-th truncation leads to the same bound as in Lemma 1.1 with $\mu$ in place of $m$ and $\varepsilon=\mu^{-1 / 2}$ for $|z| \leqslant \mu^{\beta / 2}, \beta \in(0,1 / 3)$. Note that the $P_{k}$ here are slightly different (in fact simpler): since no logarithm is taken, the cumulants $\mu_{\alpha}$ are replaced with just $\mathbb{E} X^{\alpha}$. Also for $|z| \leqslant \mu^{1 / 2} \delta=\mu^{1 / 2} \min \left\{\lambda_{1, X} / \kappa_{3, X}, \kappa_{n, X}^{-1 / n} / 2\right\}$, one still has

$$
|\psi(z)|=\left|\exp \left\{\mu\left(\chi\left(\mu^{-\frac{1}{2}} z\right)-1\right)-i \mu^{\frac{1}{2}} z \cdot \mathbb{E} X\right\}\right| \leqslant e^{-\frac{1}{2} z \cdot \Sigma_{X} z+\frac{1}{6} \mu^{-\frac{1}{2}} \mathbb{E}|X|^{3}|z|^{3}} \leqslant e^{-\frac{1}{4} z \cdot \Sigma_{X} z} .
$$

Moreover, by imposing Cramér's condition $\mathbf{C C}(\rho, \gamma)$ on the distribution of $X$, one can still achieve a similar bound for $|\psi|$ :

$$
\begin{aligned}
|\psi(z)| & =\left|\exp \left\{\chi\left(\mu^{-\frac{1}{2}} z\right)-1-i \mu^{-\frac{1}{2}} z \cdot \mathbb{E} X\right\}\right|^{\mu} \\
& =\left(\exp \left\{\operatorname{Re} \chi\left(\mu^{-\frac{1}{2}} z\right)-1\right\}\right)^{\mu} \leqslant\left(e^{\bar{\gamma}-1}\right)^{\mu} \in(0,1),
\end{aligned}
$$

for $|z|>\mu^{1 / 2} \delta$ and some $\bar{\gamma} \in(0,1)$ according to Lemma 1.2. Thus one arrives at virtually the same estimate as in Proposition [1.3, and therefore Theorem 1.7 still holds true for $\varepsilon=\mu^{-1 / 2}$ sufficiently small w.r.t. $p+2$, and Corollary 1.9 applies with $\mu$ in place of $m$ and $\exp (\bar{\gamma}-1)$ in place of $\bar{\gamma}$. For the normal approximation $(n=3)$, since no perturbing polynomials $p_{k}$ are concerned, one can scale the jumps $\widehat{X}:=\Sigma_{X}^{-1 / 2} X$ and deduce, $\forall \tau \in(0,1)$,

$$
\mathbb{W}_{p}\left(V_{1}, \mu^{\frac{1}{2}} \xi_{\Sigma_{X}}\right) \leqslant\left\|\Sigma_{X}^{\frac{1}{2}}\right\| \mathbb{W}_{p}\left(\Sigma_{X}^{-\frac{1}{2}} V_{1}, \mu^{\frac{1}{2}} \xi_{I}\right) \leqslant C_{p, q, \tau} \kappa_{p+2+\tau, \widehat{X}}^{2 \vee(p-1)^{2}} \lambda_{q, X}^{1 / 2}
$$

One can apply the above arguments to the jump process (2.2) by scaling the jump sizes. For the jumps $X_{j}^{r}$ on each annulus $\Omega_{r}$, define $X_{j}:=2^{r} X_{j}^{r}$ and $\widehat{X}_{j}:=\Sigma_{X}^{-1 / 2} X_{j}$ accordingly. For each fixed $r$, the $X_{j}^{r}$ 's are i.i.d. with characteristic function

$$
\chi^{r}(s)=\nu_{r}^{-1} \int_{\Omega_{r}} e^{i s \cdot x} \nu(\mathrm{d} x) .
$$


This implies that $X$ has scaled covariance $\Sigma_{X}=\nu_{r}^{-1} 2^{2 r} \int_{\Omega_{r}} x x^{\top} \nu(\mathrm{d} x)$ with eigenvalues $\lambda_{j, X}=$ $\nu_{r}^{-1} 2^{2 r} \lambda_{j, r}$, where $\lambda_{1, r} \leqslant \cdots \leqslant \lambda_{q, r}$ are the eigenvalues of $\Sigma_{r}:=\int_{\Omega_{r}} x x^{\top} \nu(\mathrm{d} x)$. Also notice that $\mathbb{E}|X|^{M}=\nu_{r}^{-1} 2^{r M} \int_{\Omega_{r}}|x|^{M} \nu(\mathrm{d} x) \leqslant 1, \forall M>0$, implying that $\mathbb{E}|\widehat{X}|^{M} \leqslant \lambda_{1, X}^{-M / 2}$.

Thus, if $\Sigma_{r}$ is non-singular for each $r$, then (assuming $\lambda_{1, X} \leqslant 1$ w.l.o.g.) one can apply (2.4) with parameter $\mu=t \nu_{r}$ :

$$
\mathbb{W}_{p}\left(V_{t}^{r}, \sqrt{t} \xi_{\Sigma_{r}}\right)=2^{-r} \mathbb{W}_{p}\left(2^{r} V_{t}^{r}, \sqrt{t \nu_{r}} \xi_{\Sigma_{X}}\right) \leqslant C_{p, q, \tau} \lambda_{1, X}^{-\left(1 \vee \frac{(p-1)^{2}}{2}\right)(p+2+\tau)} \lambda_{q, r}^{1 / 2} \nu_{r}^{-1 / 2} .
$$

Denote further $\Sigma_{\epsilon}:=\int_{0<|x| \leqslant \epsilon} x x^{\top} \nu(\mathrm{d} x)$, then from this bound one can find a coupling between $Z_{t}^{\epsilon}$ and $\mathcal{N}\left(0, t \Sigma_{\epsilon}\right)$ under suitable conditions.

Theorem 2.1. Suppose $\xi_{r}(s):=\chi^{r}\left(2^{r} s\right)$ satisfies $\mathbf{C C}(\rho, \gamma)$ uniformly for all $r \geqslant r_{0}$. If $\nu_{r}^{-1}=o\left(2^{-r}\right)$ as $r \rightarrow \infty$, then $\forall p \in 2 \mathbb{Z}^{+}, t \geqslant \epsilon$ and $\epsilon$ sufficiently small,

$$
\mathbb{W}_{p}\left(Z_{t}^{\epsilon}, \sqrt{t} \xi_{\Sigma_{\epsilon}}\right) \leqslant C_{p, q} \epsilon
$$

Proof. Note that on each $\Omega_{r}$ it is always true that $\lambda_{q, r} \leqslant \operatorname{tr} \Sigma_{r} \leqslant 2^{-2 r} \nu_{r}$ and $\lambda_{q, r} \geqslant q^{-1} \operatorname{tr} \Sigma_{r} \geqslant$ $q^{-1} 2^{-2(r+1)} \nu_{r}$. Write $\xi_{r}(s)=\left|\xi_{r}(s)\right| e^{i \theta}$, where $\theta=\theta(r, s)$ satisfies $\int_{\Omega_{r}} \sin \left(2^{r} s \cdot x-\theta\right) \nu(\mathrm{d} x)=0$. Then $\int_{\Omega_{r}} \sin \left(2^{r} s \cdot x\right) \nu(\mathrm{d} x)=\tan \theta \int_{\Omega_{r}} \cos \left(2^{r} s \cdot x\right) \nu(\mathrm{d} x)$ if $\theta \not \equiv \pm \pi / 2 \bmod \pi$, and otherwise $\int_{\Omega_{r}} \cos \left(2^{r} s \cdot x\right) \nu(\mathrm{d} x)=0$. By the uniform Cramér's condition for $\xi_{r}(s)$, there exist $\rho>0, \gamma \in$ $(0,1)$ s.t. $\forall r \geqslant r_{0}$ and $|s| \geqslant \rho$,

$$
\left|\xi_{r}(s)\right|=\nu_{r}^{-1} \int_{\Omega_{r}} \cos \left(2^{r} s \cdot x-\theta\right) \nu(\mathrm{d} x) \in[0, \gamma]
$$

If $\theta \not \equiv \pm \pi / 2 \bmod \pi$, expand out the integrand using the identity $\cos (\alpha-\beta)=\cos \alpha \cos \beta+$ $\sin \alpha \sin \beta, \forall \alpha, \beta \in \mathbb{R}$, replace the term $\int_{\Omega_{r}} \sin \left(2^{r} s \cdot x\right) \nu(\mathrm{d} x)$ and rearrange to get

$$
\left(\nu_{r} \cos \theta\right)^{-1} \int_{\Omega_{r}} \cos \left(2^{r} s \cdot x\right) \nu(\mathrm{d} x) \in[0, \gamma]
$$

Therefore, regardless of the values of $\theta$, one always has $\left|\int_{\Omega_{r}} \cos \left(2^{r} s \cdot x\right) \nu(\mathrm{d} x)\right| \leqslant \gamma \nu_{r}$ for $|s| \geqslant \rho$. Write $s=|s| v$ where $v \in \mathbb{S}^{q-1}$ is a unit vector. Then for $|s| \geqslant \rho$,

$$
\begin{aligned}
v \cdot \Sigma_{r} v= & \int_{\Omega_{r}}|v \cdot x|^{2} \nu(\mathrm{d} x) \geqslant 2^{-2 r+2}|s|^{-2} \int_{\Omega_{r}} \sin ^{2}\left(2^{r-1} s \cdot x\right) \nu(\mathrm{d} x) \\
& =2^{-2 r+1}|s|^{-2} \int_{\Omega_{r}}\left(1-\cos \left(2^{r} s \cdot x\right)\right) \nu(\mathrm{d} x) \geqslant 2^{-2 r+1} \rho^{-2}(1-\gamma) \nu_{r} .
\end{aligned}
$$

This means $\lambda_{1, r} \gtrsim 2^{-2 r} \nu_{r}$ by choosing $v$ to be the eigenvector of $\lambda_{1, r}$. Hence $\lambda_{1, r} \simeq \lambda_{q, r} \simeq$ $2^{-2 r} \nu_{r}$ and $\lambda_{1, X}=\nu_{r}^{-1} 2^{2 r} \lambda_{1, r} \simeq 1, \forall r \geqslant r_{0}$.

Since $\xi_{r}(s)$ is the characteristic function of $X=2^{r} X^{r}$, the uniform Cramér's condition validates the bound (2.3) with a uniform $\bar{\gamma}=\bar{\gamma}(\rho, \gamma)$ and (2.4) holds with $\mu=t \nu_{r} \geqslant \epsilon \nu_{r} \geqslant 2^{-r} \nu_{r}$ 
sufficiently large w.r.t. $p+2$. More precisely, one can choose $\epsilon$ sufficiently small s.t. for all $r \geqslant r_{0}$, similar to (1.10),

$$
\left(e^{\bar{\gamma}-1}\right)^{2^{-r} \nu_{r}}\left(2^{-r} \nu_{r}\right)^{(q+1)(p+3) / 2} \lesssim 1
$$

Thus, all the arguments leading towards (2.5) are justified, which is immediately reduced to the bound $\mathbb{W}_{p}\left(V_{t}^{r}, \sqrt{t} \xi_{\Sigma_{r}}\right) \leqslant C_{p, q} 2^{-r}$, and therefore

$$
\mathbb{W}_{p}\left(Z_{t}^{\epsilon}, \sqrt{t} \xi_{\Sigma_{\epsilon}}\right)=\mathbb{W}_{p}\left(\sum_{r=r_{0}}^{\infty} V_{t}^{r}, \sum_{r=r_{0}}^{\infty} \sqrt{t} \xi_{\Sigma_{r}}\right) \leqslant C_{p, q} \sum_{r=r_{0}}^{\infty} 2^{-r}=C_{p, q} \epsilon .
$$

Together with the finite second moment of $\nu$, the theorem above requires the order of $\nu_{r}$ is between $O\left(2^{r+}\right)$ and $O\left(2^{2 r}\right)$ as $r \rightarrow \infty$, i.e. the Lévy measure needs to be sufficiently singular near 0 . The uniform Cramér condition requires certain comparability between $\nu$ and the Lebesgue measure $\Lambda$, conditional on $\Omega_{r}$. The following lemma gives a sufficient condition.

Lemma 2.2. If there exist $a, b \in(0,1)$ s.t. for each $r \geqslant r_{0}$, any measurable subset $\Gamma_{r}$ of $\Omega_{r}$ satisfying $\Lambda\left(\Gamma_{r}\right) / \Lambda\left(\Omega_{r}\right) \geqslant a$ must have that $\nu\left(\Gamma_{r}\right) / \nu\left(\Omega_{r}\right) \geqslant b$, then $\xi_{r}(s)=\chi^{r}\left(2^{r} s\right)$ satisfies $\mathbf{C C}(\rho, \gamma)$ uniformly for all $r \geqslant r_{0}$.

Proof. For any $a^{\prime} \in(0,1)$ denote $b^{\prime}=\sin ^{2} \frac{\pi}{2}\left(1-a^{\prime}\right) \in(0,1)$. For any $\theta \in \mathbb{R}, v \in \mathbb{R}^{q}$, consider

$$
D_{k}=D_{k}(v, \theta):=\left\{x \in \Omega_{r}: 2 k \pi+\left(1-a^{\prime}\right) \pi \leqslant v \cdot x-\theta \leqslant 2(k+1) \pi-\left(1-a^{\prime}\right) \pi\right\}, k \in \mathbb{Z},
$$

on each of which $\sin ^{2} \frac{1}{2}(v \cdot x-\theta) \geqslant b^{\prime}$. They are parallel "stripes" across the annulus $\Omega_{r}$ with width $2 a^{\prime} \pi /|v|$ equidistantly away from each other by $2\left(1-a^{\prime}\right) \pi /|v|$. This can be seen by rotating so that $v$ lies on one axis. Thus for $|v|>\pi 2^{r+1}$ there is at least one non-empty $D_{k}$. Denote $\Gamma_{r}=\bigcup_{k \in \mathbb{Z}} D_{k}$, then the ratio $\Lambda\left(\Gamma_{r}\right) / \Lambda\left(\Omega_{r}\right)$ approaches $a^{\prime}$ as $|v| \rightarrow \infty$, regardless of the translation $\theta$. Therefore one can find some constants $\rho>0$ and $\gamma^{\prime}=\gamma^{\prime}(\rho, q) \in\left(0, a^{\prime}\right)$ s.t. for all $|v| \geqslant 2^{r} \rho, \Lambda\left(\Gamma_{r}\right) / \Lambda\left(\Omega_{r}\right) \geqslant \gamma^{\prime}$. Choose $\gamma^{\prime}=a$ as given in the assumption, then $\nu\left(\Gamma_{r}\right) / \nu\left(\Omega_{r}\right) \geqslant b$ for all $|v| \geqslant 2^{r} \rho$. Write $\xi_{r}(s)=\left|\xi_{r}(s)\right| e^{i \theta}$ for some $\theta=\theta(r, s)$, then

$$
1-\left|\xi_{r}(s)\right| \geqslant 2 \nu_{r}^{-1} \int_{\Gamma_{r}} \sin ^{2} \frac{1}{2}\left(2^{r} s \cdot x-\theta\right) \nu(\mathrm{d} x) \geqslant 2 b^{\prime} \nu\left(\Gamma_{r}\right) / \nu\left(\Omega_{r}\right) \geqslant 2 b^{\prime} b,
$$

for all $r \geqslant r_{0}$, and the result follows by setting $v=2^{r} s$ and $\gamma=1-2 b^{\prime} b \in(0,1)$.

Corollary 2.3. If $\nu$ satisfies $\mathbf{H}(\tau, \alpha)$ for $\alpha \in(1,2)$, then Theorem [2.1 holds for $\epsilon \in(0, \tau)$ sufficiently small.

Proof. One just needs to check that the assumptions in Theorem 2.1 are satisfied. First of all, for $\alpha \in(1,2)$ and $\forall r \geqslant r_{0}$,

$$
\nu_{r} \simeq \int_{\Omega_{r}}|x|^{-q-\alpha} \mathrm{d} x=C_{q} \frac{2^{\alpha}-1}{\alpha} 2^{\alpha r} .
$$


Then $2^{r} \nu_{r}^{-1}=o(1)$ for $\alpha \in(1,2)$. For any measurable subset $\Gamma_{r}$ of $\Omega_{r}$,

$$
\frac{\nu\left(\Gamma_{r}\right)}{\nu\left(\Omega_{r}\right)} \geqslant \frac{\int_{\Gamma_{r}}|x|^{-q-\alpha} \mathrm{d} x}{\int_{\Omega_{r}}|x|^{-q-\alpha} \mathrm{d} x} \geqslant 2^{-q-\alpha} \frac{\Lambda\left(\Gamma_{r}\right)}{\Lambda\left(\Omega_{r}\right)},
$$

which validates Lemma 2.2 .

It is worth mentioning that if the condition $\mathbf{H}(\tau, \alpha)$ is assumed a priori, then one could directly use the Lévy-Khintchine formula to study the global behaviour of the characteristic function of $Z_{t}^{\epsilon}$, which would greatly simplify the analysis of Section 1, but the same arguments used in the proof of Theorem 1.7 would still be needed for the coupling result.

\subsection{A Coupling for Euler's Approximation}

Given the coupling result above, one finally arrives at the stage of recovering Fournier's results [9] on the Euler approximation of the SDE (0.2) driven by the Lévy process (0.1):

$$
\begin{aligned}
& x_{t}=x_{0}+\int_{0}^{t} \sigma\left(x_{s-}\right) \mathrm{d} Z_{s}, t \in[0, T], \\
& Z_{t}=a t+B W_{t}+\int_{0}^{t} \int_{\mathbb{R}^{q} \backslash\{0\}} z \widetilde{N}(\mathrm{~d} z, \mathrm{~d} s) .
\end{aligned}
$$

For fixed $h, \epsilon \in(0,1)$ introduce the following random variable

$$
\bar{\Delta}_{1}:=\bar{a} h+\bar{B} \sqrt{h} \xi_{I}+\sum_{j=1}^{N_{h}^{\epsilon}} Y_{j}^{\epsilon},
$$

and take independent copies $\bar{\Delta}_{2}, \cdots, \bar{\Delta}_{[T / h]}$, where $\left\{Y_{j}^{\epsilon}\right\}$ are i.i.d. random variables having distribution $\mathbb{1}_{|z|>\epsilon} \nu(\mathrm{d} z) / \nu(|z|>\epsilon), N_{h}^{\epsilon}$ is Poisson with parameter $h \nu(\{|z|>\epsilon\})$, and the coefficients $\bar{a}=a-\int_{|z|>\epsilon} z \nu(\mathrm{d} z), \bar{B}:=\left(B B^{\top}+\Sigma_{\epsilon}\right)^{1 / 2}, \Sigma_{\epsilon}=\int_{0<|z| \leqslant \epsilon} z z^{\top} \nu(\mathrm{d} z)$. For $t_{k}=$ $k h, k=1, \cdots,[T / h]$, write the increments $\Delta_{k}:=Z_{t_{k}}-Z_{t_{k-1}}$. Then one may attempt to find a coupling between the standard Euler's approximation

$$
X_{k+1}:=X_{k}+\sigma\left(X_{k}\right) \Delta_{k+1}, X_{0}=x_{0},
$$

and the numerical scheme

$$
\bar{X}_{k+1}:=\bar{X}_{k}+\sigma\left(\bar{X}_{k}\right) \bar{\Delta}_{k+1}, \bar{X}_{0}=x_{0} .
$$

For that one claims the following statement as an analogue to Lemma 5.2 in [9]:

Proposition 2.4. If $\nu$ satisfies the conditions of Theorem [2.1, then for $\epsilon$ sufficiently small there exist on the same probability space two sequences of i.i.d. random variables $\left\{\Delta_{k}^{\prime}\right\},\left\{\bar{\Delta}_{k}^{\prime}\right\}$, with the same distributions as $\left\{\Delta_{k}\right\}$ and $\left\{\bar{\Delta}_{k}\right\}$ respectively, s.t.

$$
\left(\mathbb{E}\left|\Delta_{k}^{\prime}-\bar{\Delta}_{k}^{\prime}\right|^{p}\right)^{\frac{1}{p}} \leqslant C_{q} \epsilon
$$

for all $k \in \mathbb{Z}^{+}$and $p \in 2 \mathbb{Z}^{+}$, and $\mathbb{E} \Delta_{k}^{\prime}=\mathbb{E} \bar{\Delta}_{k}^{\prime}=a h, \operatorname{var} \Delta_{k}^{\prime}=\operatorname{var} \bar{\Delta}_{k}^{\prime}=\left(B B^{\top}+\Sigma_{\epsilon}\right) h$. 
Proof. By Theorem 2.1, for $\epsilon$ sufficiently small there is a standard normal random variable $\xi_{I}^{\prime}$ on the same probability space s.t.

$$
\left(\mathbb{E}\left|\int_{0}^{h} \int_{0<|z| \leqslant \epsilon} z \widetilde{N}(\mathrm{~d} z, \mathrm{~d} s)-h \Sigma_{\epsilon}^{\frac{1}{2}} \xi_{I}^{\prime}\right|^{p}\right)^{\frac{1}{p}} \leqslant C_{q} \epsilon
$$

according to the definition of the $\mathbb{W}_{p}$ distance. If one sets

$$
\begin{aligned}
& \Delta_{1}^{\prime}:=a h+B W_{h}+\int_{0}^{h} \int_{0<|z| \leqslant \epsilon} z \widetilde{N}(\mathrm{~d} z, \mathrm{~d} s)+\int_{0}^{h} \int_{|z|>\epsilon} z \tilde{N}(\mathrm{~d} z, \mathrm{~d} s), \\
& \bar{\Delta}_{1}^{\prime}:=a h+B W_{h}+h \Sigma_{\epsilon}^{\frac{1}{2}} \xi_{I}^{\prime}+\int_{0}^{h} \int_{|z|>\epsilon} z \tilde{N}(\mathrm{~d} z, \mathrm{~d} s),
\end{aligned}
$$

then $\Delta_{1}^{\prime}$ has the same law as $\Delta_{1}$, and $\bar{\Delta}_{1}^{\prime}$ has the same law as $\bar{\Delta}_{1}$. Thus the result follows by taking independent copies.

Proposition 2.4 can be immediately used to partially recover the main results in [9] (Theorem 2.2): the proof is independent of the key coupling result (Lemma 5.2), so one can replace the latter with the proposition above. Hence one can restate those results as follows:

Theorem 2.5. Suppose $\sigma: \mathbb{R}^{d} \rightarrow \mathbb{R}^{d \times q}$ is bounded and Lipschitz, and the Lévy measure $\nu$ for the Lévy process (2.7) satisfies conditions of Theorem [2.1. Let $\epsilon, h \in(0,1)$, and $\left\{x_{t}\right\}$ be the unique solution to the $S D E(2.6)$ for $t \in[0, T]$. Then for $\rho_{h}(t)=[t / h] h$ and $\epsilon$ sufficiently small, there exists a coupling between $\left\{x_{t}\right\}$ and $\left\{\bar{X}_{\rho_{n}(t)}\right\}$ defined by (2.9) and (2.8) s.t.

$$
\mathbb{E} \sup _{t \in[0, T]}\left|x_{t}-\bar{X}_{\rho_{h}(t)}\right|^{2} \leqslant C_{1}(h+\epsilon) .
$$

Moreover, if $\nu(\{|z|>\epsilon\})=0$, i.e. $Z_{t}=Z_{t}^{\epsilon}$ as in (2.7), and $\left\{\widetilde{x}_{t}^{\epsilon}\right\}$ is the unique solution to the continuous $S D E \widetilde{x}_{t}^{\epsilon}=x_{0}+\int_{0}^{t} \sigma\left(\widetilde{x}_{t}^{\epsilon}\right) \mathrm{d} \widetilde{Z}_{t}^{\epsilon}$ where $\widetilde{Z}_{t}^{\epsilon}=a t+\left(B B^{\top}+\Sigma_{\epsilon}\right)^{1 / 2} W_{t}$, then there exists a coupling between $x_{t}$ and $\widetilde{x}_{t}^{\epsilon}$ s.t.

$$
\mathbb{E} \sup _{t \in[0, T]}\left|x_{t}-\widetilde{x}_{t}^{\epsilon}\right|^{2} \leqslant C_{2} \epsilon
$$

The constants $C_{1}, C_{2}$ depend on $d, q, T,|a|,\|B\|,\|\sigma\|_{\infty}, \Sigma_{\epsilon}$.

Instead of repeating the same arguments of Fournier [9], the reader is referred to the proof of Theorem 2.2 therein. Note that Proposition 2.4 above allows one to replace the $\beta_{\epsilon}(\nu)$ in Lemma 5.2 with $\epsilon^{2}$, and the rest of the calculations can be readily generalised to the multi-dimensional case. In particular, under the assumption $\mathbf{H}(\tau, \alpha)$ for some $\alpha \in(1,2)$, by choosing $\epsilon=h$ one recovers the mean-square convergence rate $O(h)$ and the computational cost $E_{\nu}(h)=O\left(h^{-1}+h^{-\alpha}\right)$ is controlled. The second statement corresponds to Corollary 3.2 in [9]. For that, one simply takes $\bar{\Delta}_{1}=\bar{a} h+\bar{B} \sqrt{h} \xi_{I}$ instead of (2.8) and $h=\epsilon$, and runs the same argument as in Proposition 2.4, omitting the big-jump part.

The general case where $\sigma$ is locally Lipschitz with linear growth and only $\int_{\mathbb{R}^{q} \backslash\{0\}} 1 \wedge|z|^{2} \nu(\mathrm{d} z)<$ $\infty$ is assumed can be treated by the same localisation argument as in Theorem 7.1 in [9], and 
the mean-square convergence could be generalised to the strong $L^{p}$-convergence for $p \in 2 \mathbb{Z}^{+}$ without much trouble. Nevertheless, it needs to be pointed out that the rate of convergence here is optimal for coupling the small jumps only - it might not be so if one can couple the entire Lévy increment. For the same reason the results achieved in this article cannot be applied to recover Theorem 3.1 in [9. Finally, I believe the conditions of Theorem 2.1 can be relaxed to some extent. E.g., one may take a hint from Proposition A.2 in [10] that it possibly suffices for $\nu$ to give a suitable portion of mass to the biggest annulus $\Omega_{r_{0}}$.

Acknowledgement. I am grateful for the patient guidance of my Ph.D. supervisor, Prof. A. M. Davie, who suggested these problems and gave many helpful comments. I would also like to thank the referees for drawing my attention to several related works that I was not aware of, particularly [3], [5] and [10].

\section{References}

[1] Asmussen, S., And Rosiński, J. Approximations of small jumps of Lévy processes with a view towards simulation. Journal of Applied Probability 38, 2 (June 2001), 482-493.

[2] Bhattacharya, R. N., And RaO, R. R. Normal Approximation and Asymptotic Expansions. Wiley Series in Probability and Mathematical Statistics. John Wiley \& Sons, 1976.

[3] Bobkov, S. G. Berry-Esseen bounds and edgeworth expansions in the central limit theorem for transport distances. Probability Theory and Related Fields (2017).

[4] Bobkov, S. G., Chistyakov, G. P., and Götze, F. Rate of convergence and Edgeworth-type expansion in the entropic central limit theorem. The Annals of Probability 41, 4 (2013), 2479-2512.

[5] Bonis, T. Rates in the central limit theorem and diffusion approximation via Stein's method. arXiv:1506.06966v3 (2016).

[6] Davie, A. M. Pathwise approximation of stochastic differential equations using coupling. Preprint (2014), www.maths.ed.ac.uk/ sandy/coum.pdf.

[7] Davie, A. M. Polynomial perturbations of normal distributions. Preprint (2016), www.maths.ed.ac.uk/ sandy/polg.pdf.

[8] Einmahl, U. Extensions of results of Komlós, Major, And Tusnády to the multivariate case. Journal of Multivariate Analysis 28 (1989), 20-68.

[9] Fournier, N. Simulation and approximation of Lévy-driven stochastic differential equations. ESAIM. Probability and Statistics 15 (2011), 233-248.

[10] Godinho, D. Asymptotic of grazing collisions for the spatially homogeneous Boltzmann equations for soft and Coulomb potentials. Stochastic Processes and Their Applications 123 (2013), 3987-4039. 
[11] Götze, F., And ZAitsev, A. Y. Rates of approximation in the multidimensional invariance principle for sums of i.i.d. random vectors with finite moments. Journal of Mathematical Sciences 167, 4 (2010).

[12] JACOD, J. The Euler scheme for Lévy driven stochastic differential equations: Limit theorems. The Annals of Probability 32, 3A (2004), 1830-1872.

[13] Jacod, J., And Protter, P. Asymptotic error distributions for the Euler method for stochastic differential equations. The Annals of Probability 26, 1 (1998), 267-307.

[14] Kohatsu-Higa, A., And Protter, P. The Euler scheme for SDEs driven by semimartingales. Stochastic Analysis on Infinite Dimensional Spaces (1994), 141-151.

[15] Komlós, J., Major, P., And Tusnády, G. An approximation of partial sums of independent RV'-s, and the sample of DF.I. Z. Wahrscheinlichkeitstheorie Verw Gebiete 32 (1975), 111-131.

[16] Petrov, V. V. Sums of Independent Random Variables. Springer-Verlag, 1975.

[17] RIO, E. Upper bounds for minimal distances in the central limit theorem. Annales de l'Institut Henri Poincaré - Probabilités et Statistiques 45, 3 (2009), 802-817.

[18] RIo, E. Asymptotic constants for minimal distances in the central limit theorem. Elect. Comm. in Probab. 16 (Dec. 2011).

[19] Talagrand, M. Transportation cost for Gaussian and other product measures. Geometric and Functional Analysis 6, 3 (1996).

[20] Villani, C. Topics in Optimal Transportation, vol. 58. American Mathematical Society, 2003.

[21] Zaitsev, A. Y. Multidimensional version of the results of Komlós, Major and Tusnády for vectors with finite exponential moments. ESAIM. Probability and Statistics 2 (1998), 41-108.

[22] Zaitsev, A. Y. Estimates for the strong approximation in multidimensional central limit theorem. Proceedings of the International Congress of Mathematicians III, Beijing (2002), 107-116. 